\title{
Effect of Graded Levels of NPK Fertilizers on Pests Incidence in Bt Cotton in Alfisol
}

\author{
T.V. Jyothi ${ }^{1 *}$, N.S. Hebsur ${ }^{2}$ and Parashuram Chandravanshi ${ }^{3}$
}

${ }^{1}$ Krishi Vigyan Kendra, Babbur farm, Hiriyur, UAHS, Shivamogga, India

${ }^{2}$ Department of Soil Science and Agricultural Chemistry, University of Agricultural Sciences, Dharwad-580005, Karnataka, India

${ }^{3}$ ZAHRS, Babbur farm, Hiriyur, UAHS, Shivamogga, Karnataka, India

*Corresponding author

\section{A B S T R A C T}

\begin{tabular}{|l|}
\hline K e y w o r d s \\
Alfisol, Cotton, \\
Fertilizer, Nutrient, \\
pest
\end{tabular}

\section{Introduction}

Cotton is an important commercial crop unanimously designated as 'king of fibre crops' and is prone to insect pests attack at various stages of crop growth. Compared to world average cotton lint yield $\left(600 \mathrm{~kg} \mathrm{ha}^{-1}\right)$, India produces around $375 \mathrm{~kg}$ lint ha ${ }^{-1}$. The low cotton lint yield is associated because of number of reasons, of them, its cultivation under rainfed situation and pest infestation. Introduction of synthetic pyrethroids, though brought desirable control of bollworms, resulted in resurgence of sucking pests viz., aphid ( Aphis gossypii Glover), leafhopper
[Amrasca biguttula biguttula (Ishida)], thrips (Thrips tabaci Lindeman) and whitefly [Bemisia tabaci (Gennadius)] (Ajri et al., 1986 and Patil et al., 1986). In the last few decades, these pests became very serious pests of cotton and many other crop plants in tropical and subtropical areas of the world (El-Zahi et al., 2012). Nutrient management improves the plant health, which enables the plant to tolerate against the incidence and attack of herbivores.

Fertilizers, especially nitrogen fertilizer, are the major factors to increase crop yield, and can influence pest populations by reducing 
plant resistance to insects (Altieri and Nicholls, 2003; Way et al., 2006). Previous studies have showed that increased nitrogen supply is related to the occurrence of insect herbivores such as Aphis gossypii in cotton (Nevo and Coll, 2001), Liriomyza trifolii (Facknath and Lalljee, 2005) and whitefly (Bi et al., 2001). Therefore, it is widely accepted that many crops supplied with nitrogen fertilizer are favorable for many herbivore insects, despite of their promoting crop growth and yield. Potassium has been considered to be a key component of plant nutrition that significantly influences crop growth and some pests' infestation. A prevailing view is that a high potassium status in plant tissues supplied by soil decreases the incidence of many pests. Potassium fertilizer is negatively associated with occurrence of $L$. trifolii (Facknath and Lalljee, 2005), Aphis glycines (Myers and Gratton, 2006), leafhoppers and mites (Parihar and Upadhyay, 2001).

Therefore, an understanding of basic agronomic practices such as optimal row spacing, fertilizer rates, insect pests, diseases and crop response to these factors are essential for maximizing yields. Sufficient nutrient supply and successful protection of the crop against herbivores and pathogens are critical for crop yield and quality in modern agriculture (Amtmann et al., 2008). Keeping all these points in view, a research work was framed with an objective of studying the effects of nitrogen, phosphorus and potassium fertilizers either alone or in combinations on the population densities of pests.

\section{Materials and Methods}

A field experiment was conducted in farmer's field one at Jodalli village (Kalghatgi taluk) in 2012-13 situated at $15^{\circ} 19^{\prime} 865^{\prime \prime}$ North latitude and $75^{\circ} 00^{\prime} 65^{\prime \prime}$ East longitude and another at Pale village (Hubballi taluk) in 2013-14 situated at $15^{0} 14^{\prime} 404^{\prime \prime}$ North latitude and $75^{\circ} 08^{\prime} 600^{\prime \prime}$ East longitude under protective irrigated condition to find out the appropriate NPK levels for $\mathrm{Bt}$ cotton in Alfisol. The farmer of Jodalli village did not agree to take up the experiment during second year. Hence, the experiment was conducted at Pale village. The spacing adopted was $90 \mathrm{~cm}$ and between rows and $60 \mathrm{~cm}$ between plants for hybrid cotton. The factorial randomized complete block Design with nineteen treatments and three replications was adopted. The treatment details are given below.

\section{Treatment details}

\section{A. Factor - I (N levels)}

$\mathrm{N}_{1}: 100 \mathrm{~kg} \mathrm{ha}^{-1}, \mathrm{~N}_{2}: 125 \mathrm{~kg} \mathrm{ha}^{-1}, \mathrm{~N}_{3:} 150 \mathrm{~kg} \mathrm{ha}^{-}$

\section{B. Factor - II ( $\mathrm{P}_{2} \mathrm{O}_{5}$ levels $)$}

$\mathrm{P}_{1}: 50 \mathrm{~kg} \mathrm{ha}^{-1}, \mathrm{P}_{2}: 75 \mathrm{~kg} \mathrm{ha}^{-1}$

\section{Factor - III ( $\mathrm{K}_{2} \mathrm{O}$ levels $)$}

$\mathrm{K}_{1}: 50 \mathrm{~kg} \mathrm{ha}^{-1}, \mathrm{~K}_{2}: 75 \mathrm{~kg} \mathrm{ha}^{-1}, \mathrm{~K}_{3}: 100 \mathrm{~kg} \mathrm{ha}^{-1}$

\section{Absolute control}

Entire recommended dose of phosphorus and potassium and 50 per cent of nitrogen were applied after germination by ring method. Remaining 50 per cent of nitrogen was applied at 60 DAS as per the package of practice. Adequate plant protection measures were taken as per the recommended package for Bt cotton as and when required at various growth stages commonly to all the treatments. The plant protection measures for the control of sucking pests (thrips, jassids, aphids, shoot weevil, mirid bug and midge) were taken as and when required at various growth stages commonly to all the treatments. 


\section{Scoring of pests}

Observations were made on thrips, jassids and aphids on three leaves (top, middle and bottom), shoot weevil (10 random plants), mirid bug and midge (10 squares) from each of 10 randomly selected plants from each plot. The incidence of pest was recorded by using 1-4 grade (Kranthi et al., 2009) and the observations were then converted to transformed values.

\section{Results and Discussion}

\section{Effect of different levels of NPK fertilizers on pests population in Bt cotton}

The pooled data revealed that, sucking pests populations were significantly affected by different levels of nitrogen and potassium application. Significantly higher thrips, jassids, aphids and shoot weevil populations (2.58 and 1.68, 2.14 and 1.55, 3.49 and 2.37 per 3 leaves and 4.03 and 3.63 per 10 plants) were recorded in the treatment $\mathrm{N}_{3}(150 \mathrm{~kg} \mathrm{~N}$ $\mathrm{ha}^{-1}$ ) at 70 and 90 DAS, respectively. Ahmed et al., (2007) found that the highest rate of nitrogen resulted in the highest per leaf mean population of jassids, whitefly and thrips. $\mathrm{He}$ reported that, an excessive dose of nitrogen fertilizer might produce lush green plants, which will attract pests. Cisneros and Godfery (1998) reported that nitrogen affected the population dynamics of naturally occurring aphids with higher densities in plots receiving high $\mathrm{N}$ rates. Godfery et al., (1999) mentioned that high levels of nitrogen fertilization appear to promote increased cotton aphid reproduction and the build-up of high in field aphid populations.

Different levels of phosphorus showed significant effect on jassids and shoot weevil population (1.41 per 3 leaves at 90 DAS and 3.84 and 3.59 per 10 plants at 70 and 90 DAS, respectively). In case of potassium levels, with the increase in levels of potassic fertilizers there was a decrease in pest population. The treatment receiving K @ 100 $\mathrm{kg} \mathrm{ha}^{-1}\left(\mathrm{~K}_{3}\right)$ recorded lower thrips, jassids, aphids and shoot weevil populations (2.29 and $1.40,1.88$ and $1.23,3.03$ and 2.05 per 3 leaves and 3.65 and 3.32 per 10 plants at 70 and 90 DAS, respectively) compared to other two levels.

The interaction of NP levels showed significant effect on thrips and aphids population. Significantly higher thrips and aphids population were recorded in the treatment $\mathrm{N}_{3} \mathrm{P}_{2}$ (2.64 and 1.73 and 3.56 and 2.44 per 3 leaves at 70 and 90 DAS, respectively). But, the jassids and shoot weevil populations were unaffected by the combined effect of NP levels. There was an inverse relationship found with NK interaction effect. It was observed that, increased levels of nitrogen recorded higher sucking pests incidence and incase of potassium levels it was vice versa. El-Zahi $e t$ al., (2012) in his study reported that plants fertilized with potassium either alone or in combinations with others were infested with the lowest population densities of jassids (Impoasca spp.) and aphids (Aphis gossypii). Potassium fertilizer significantly decreased the aphid population density and reduced the infestation level of cotton plants with aphids.

The combined effect of NPK fertilizers among the treatments was statistically non significant during first and second years of experimentation and in pooled data. Many studies have been done on the effect of nitrogen and potassium rates on the population density of sucking pests, but no information are available at present on the effect of combined application of nitrogen, phosphorus and potassium (Purohit and Deshpande, 1991) (Table 1-5). 
Table.1 Thrips population (per 3 leaves) in Bt cotton as influenced by different levels of NPK in Alfisol

\begin{tabular}{|c|c|c|c|c|c|c|}
\hline \multirow[t]{3}{*}{ Treatments } & \multicolumn{6}{|c|}{ Thrips (per 3 leaves) } \\
\hline & \multicolumn{3}{|c|}{70 DAS } & \multicolumn{3}{|c|}{90 DAS } \\
\hline & 2012-13 & 2013-14 & Pooled & 2012-13 & 2013-14 & Pooled \\
\hline $\mathbf{N}_{1}$ & $\begin{array}{c}2.38 \\
(1.70)\end{array}$ & $\begin{array}{c}2.43 \\
(1.71)\end{array}$ & $\begin{array}{c}2.40 \\
(1.70)\end{array}$ & $\begin{array}{c}1.40 \\
(1.38)\end{array}$ & $\begin{array}{c}1.56 \\
(1.44)\end{array}$ & $\begin{array}{c}1.48 \\
(1.41)\end{array}$ \\
\hline $\mathbf{N}_{2}$ & $\begin{array}{c}2.49 \\
(1.73)\end{array}$ & $\begin{array}{c}2.53 \\
(1.74)\end{array}$ & $\begin{array}{c}2.51 \\
(1.73)\end{array}$ & $\begin{array}{c}1.56 \\
(1.43)\end{array}$ & $\begin{array}{c}1.60 \\
(1.45)\end{array}$ & $\begin{array}{c}1.58 \\
(1.44)\end{array}$ \\
\hline $\mathbf{N}_{3}$ & $\begin{array}{c}2.56 \\
(1.75)\end{array}$ & $\begin{array}{c}2.61 \\
(1.76)\end{array}$ & $\begin{array}{c}2.58 \\
(1.76)\end{array}$ & $\begin{array}{c}1.67 \\
(1.47)\end{array}$ & $\begin{array}{c}1.69 \\
(1.48)\end{array}$ & $\begin{array}{c}1.68 \\
(1.47)\end{array}$ \\
\hline S.Em. \pm & 0.005 & 0.006 & 0.004 & 0.007 & 0.006 & 0.005 \\
\hline C.D. at $5 \%$ & 0.013 & 0.016 & 0.011 & 0.019 & 0.017 & 0.013 \\
\hline $\mathbf{P}_{1}$ & $\begin{array}{c}2.46 \\
(1.72)\end{array}$ & $\begin{array}{c}2.50 \\
(1.73)\end{array}$ & $\begin{array}{c}2.48 \\
(1.72)\end{array}$ & $\begin{array}{c}1.53 \\
(1.42)\end{array}$ & $\begin{array}{c}1.59 \\
(1.45)\end{array}$ & $\begin{array}{c}1.56 \\
(1.43)\end{array}$ \\
\hline $\mathbf{P}_{2}$ & $\begin{array}{c}2.48 \\
(1.73)\end{array}$ & $\begin{array}{c}2.55 \\
(1.74)\end{array}$ & $\begin{array}{c}2.52 \\
(1.74)\end{array}$ & $\begin{array}{c}1.55 \\
(1.43)\end{array}$ & $\begin{array}{c}1.64 \\
(1.46)\end{array}$ & $\begin{array}{c}1.60 \\
(1.45)\end{array}$ \\
\hline S.Em. \pm & 0.004 & 0.005 & 0.003 & 0.006 & 0.005 & 0.004 \\
\hline C.D. at $5 \%$ & NS & NS & NS & NS & NS & NS \\
\hline $\mathbf{K}_{1}$ & $\begin{array}{c}2.65 \\
(1.77)\end{array}$ & $\begin{array}{c}2.76 \\
(1.81)\end{array}$ & $\begin{array}{c}2.71 \\
(1.79)\end{array}$ & $\begin{array}{c}1.72 \\
(1.49)\end{array}$ & $\begin{array}{c}1.85 \\
(1.53)\end{array}$ & $\begin{array}{c}1.79 \\
(1.51)\end{array}$ \\
\hline $\mathbf{K}_{2}$ & $\begin{array}{c}2.48 \\
(1.73)\end{array}$ & $\begin{array}{c}2.52 \\
(1.74)\end{array}$ & $\begin{array}{c}2.50 \\
(1.73)\end{array}$ & $\begin{array}{c}1.53 \\
(1.42)\end{array}$ & $\begin{array}{c}1.58 \\
(1.44)\end{array}$ & $\begin{array}{c}1.55 \\
(1.43)\end{array}$ \\
\hline $\mathbf{K}_{\mathbf{3}}$ & $\begin{array}{c}2.29 \\
(1.67)\end{array}$ & $\begin{array}{c}2.29 \\
(1.67)\end{array}$ & $\begin{array}{c}2.29 \\
(1.67)\end{array}$ & $\begin{array}{c}1.38 \\
(1.37)\end{array}$ & $\begin{array}{c}1.42 \\
(1.39)\end{array}$ & $\begin{array}{c}1.40 \\
(1.38)\end{array}$ \\
\hline S.Em. \pm & 0.005 & 0.006 & 0.004 & 0.007 & 0.006 & 0.005 \\
\hline C.D. at $5 \%$ & 0.013 & 0.016 & 0.011 & 0.019 & 0.017 & 0.013 \\
\hline $\mathbf{N}_{1} \mathbf{P}_{1}$ & $\begin{array}{c}2.37 \\
(1.69)\end{array}$ & $\begin{array}{c}2.40 \\
(1.70)\end{array}$ & $\begin{array}{c}2.38 \\
(1.70)\end{array}$ & $\begin{array}{c}1.38 \\
(1.37)\end{array}$ & $\begin{array}{c}1.55 \\
(1.43)\end{array}$ & $\begin{array}{c}1.47 \\
(1.40)\end{array}$ \\
\hline $\mathbf{N}_{1} \mathbf{P}_{2}$ & $\begin{array}{c}2.39 \\
(1.70)\end{array}$ & $\begin{array}{c}2.46 \\
(1.72)\end{array}$ & $\begin{array}{c}2.42 \\
(1.71)\end{array}$ & $\begin{array}{c}1.41 \\
(1.38)\end{array}$ & $\begin{array}{c}1.57 \\
(1.44)\end{array}$ & $\begin{array}{c}1.49 \\
(1.41)\end{array}$ \\
\hline $\mathbf{N}_{2} \mathbf{P}_{1}$ & $\begin{array}{c}2.50 \\
(1.73)\end{array}$ & $\begin{array}{c}2.54 \\
(1.74)\end{array}$ & $\begin{array}{c}2.52 \\
(1.74)\end{array}$ & $\begin{array}{c}1.57 \\
(1.44)\end{array}$ & $\begin{array}{c}1.63 \\
(1.46)\end{array}$ & $\begin{array}{c}1.60 \\
(1.45)\end{array}$ \\
\hline $\mathbf{N}_{2} \mathbf{P}_{2}$ & $\begin{array}{c}2.47 \\
(1.72)\end{array}$ & $\begin{array}{c}2.51 \\
(1.73)\end{array}$ & $\begin{array}{c}2.49 \\
(1.73)\end{array}$ & $\begin{array}{c}1.55 \\
(1.43)\end{array}$ & $\begin{array}{c}1.58 \\
(1.44)\end{array}$ & $\begin{array}{c}1.56 \\
(1.43)\end{array}$ \\
\hline $\mathbf{N}_{3} \mathbf{P}_{1}$ & $\begin{array}{c}2.52 \\
(1.74)\end{array}$ & $\begin{array}{c}2.55 \\
(1.74)\end{array}$ & $\begin{array}{c}2.53 \\
(1.74)\end{array}$ & $\begin{array}{c}1.64 \\
(1.46)\end{array}$ & $\begin{array}{c}1.60 \\
(1.45)\end{array}$ & $\begin{array}{c}1.62 \\
(1.45)\end{array}$ \\
\hline $\mathbf{N}_{3} \mathbf{P}_{2}$ & $\begin{array}{c}2.60 \\
(1.76)\end{array}$ & $\begin{array}{c}2.67 \\
(1.78)\end{array}$ & $\begin{array}{c}2.64 \\
(1.77)\end{array}$ & $\begin{array}{c}1.69 \\
(1.48)\end{array}$ & $\begin{array}{c}1.78 \\
(1.51)\end{array}$ & $\begin{array}{c}1.73 \\
(1.49)\end{array}$ \\
\hline S.Em. \pm & 0.006 & 0.008 & 0.006 & 0.010 & 0.009 & 0.006 \\
\hline C.D. at $5 \%$ & 0.018 & 0.023 & 0.016 & NS & 0.025 & 0.019 \\
\hline $\mathrm{N}_{1} \mathrm{~K}_{1}$ & $\begin{array}{c}2.53 \\
(1.74)\end{array}$ & $\begin{array}{c}2.68 \\
(1.78)\end{array}$ & $\begin{array}{c}2.60 \\
(1.76)\end{array}$ & $\begin{array}{c}1.47 \\
(1.40)\end{array}$ & $\begin{array}{c}1.71 \\
(1.49)\end{array}$ & $\begin{array}{c}1.59 \\
(1.45)\end{array}$ \\
\hline $\mathbf{N}_{1} \mathbf{K}_{2}$ & $\begin{array}{c}2.42 \\
(1.71)\end{array}$ & $\begin{array}{c}2.44 \\
(1.71)\end{array}$ & $\begin{array}{c}2.43 \\
(1.71)\end{array}$ & $\begin{array}{c}1.42 \\
(1.38)\end{array}$ & $\begin{array}{c}1.53 \\
(1.42)\end{array}$ & $\begin{array}{c}1.47 \\
(1.40)\end{array}$ \\
\hline $\mathbf{N}_{1} \mathbf{K}_{3}$ & $\begin{array}{c}2.19 \\
(1.64)\end{array}$ & $\begin{array}{c}2.17 \\
(1.63)\end{array}$ & $\begin{array}{c}2.18 \\
(1.64)\end{array}$ & $\begin{array}{c}1.31 \\
(1.34)\end{array}$ & $\begin{array}{c}1.45 \\
(1.39)\end{array}$ & $\begin{array}{c}1.38 \\
(1.37)\end{array}$ \\
\hline $\mathbf{N}_{2} \mathbf{K}_{1}$ & $\begin{array}{c}2.69 \\
(1.79)\end{array}$ & $\begin{array}{c}2.78 \\
(1.81)\end{array}$ & $\begin{array}{c}2.74 \\
(1.80)\end{array}$ & $\begin{array}{c}1.78 \\
(1.51)\end{array}$ & $\begin{array}{c}1.86 \\
(1.54)\end{array}$ & $\begin{array}{c}1.82 \\
(1.52)\end{array}$ \\
\hline $\mathbf{N}_{2} \mathbf{K}_{2}$ & $\begin{array}{c}2.51 \\
(1.73)\end{array}$ & $\begin{array}{c}2.56 \\
(1.75)\end{array}$ & $\begin{array}{c}2.53 \\
(1.74)\end{array}$ & $\begin{array}{c}1.54 \\
(1.43)\end{array}$ & $\begin{array}{c}1.54 \\
(1.43)\end{array}$ & $\begin{array}{c}1.54 \\
(1.43)\end{array}$ \\
\hline $\mathbf{N}_{2} \mathbf{K}_{3}$ & $\begin{array}{c}2.26 \\
(1.66)\end{array}$ & $\begin{array}{c}2.24 \\
(1.66)\end{array}$ & $\begin{array}{c}2.25 \\
(1.66)\end{array}$ & $\begin{array}{c}1.37 \\
(1.37)\end{array}$ & $\begin{array}{c}1.41 \\
(1.38)\end{array}$ & $\begin{array}{c}1.39 \\
(1.37)\end{array}$ \\
\hline $\mathbf{N}_{3} \mathbf{K}_{1}$ & $\begin{array}{c}2.73 \\
(1.80)\end{array}$ & $\begin{array}{c}2.83 \\
(1.82)\end{array}$ & $\begin{array}{c}2.78 \\
(1.81)\end{array}$ & $\begin{array}{c}1.90 \\
(1.55)\end{array}$ & $\begin{array}{c}1.99 \\
(1.58)\end{array}$ & $\begin{array}{c}1.95 \\
(1.56)\end{array}$ \\
\hline $\mathbf{N}_{3} \mathbf{K}_{2}$ & $\begin{array}{c}2.51 \\
(1.73)\end{array}$ & $\begin{array}{c}2.56 \\
(1.75)\end{array}$ & $\begin{array}{c}2.53 \\
(1.74)\end{array}$ & $\begin{array}{c}1.62 \\
(1.46)\end{array}$ & $\begin{array}{c}1.66 \\
(1.47)\end{array}$ & $\begin{array}{c}1.64 \\
(1.46)\end{array}$ \\
\hline $\mathbf{N}_{3} \mathbf{K}_{3}$ & $\begin{array}{c}2.43 \\
(1.71)\end{array}$ & $\begin{array}{c}2.45 \\
(1.72)\end{array}$ & $\begin{array}{c}2.44 \\
(1.71)\end{array}$ & $\begin{array}{c}1.48 \\
(1.41)\end{array}$ & $\begin{array}{c}1.42 \\
(1.38)\end{array}$ & $\begin{array}{c}1.45 \\
(1.39)\end{array}$ \\
\hline S.Em. \pm & 0.008 & 0.010 & 0.007 & 0.012 & 0.011 & 0.008 \\
\hline C.D. at $5 \%$ & 0.023 & 0.028 & 0.020 & 0.034 & 0.030 & 0.023 \\
\hline
\end{tabular}


Contd.

\begin{tabular}{|c|c|c|c|c|c|c|}
\hline \multirow[t]{3}{*}{ Treatments } & \multicolumn{6}{|c|}{ Thrips (per 3 leaves) } \\
\hline & \multicolumn{3}{|c|}{70 DAS } & \multicolumn{3}{|c|}{90 DAS } \\
\hline & 2012-13 & 2013-14 & Pooled & 2012-13 & 2013-14 & Pooled \\
\hline $\mathbf{P}_{1} \mathbf{K}_{1}$ & $\begin{array}{c}2.65 \\
(1.78)\end{array}$ & $\begin{array}{c}2.75 \\
(1.80)\end{array}$ & $\begin{array}{c}2.70 \\
(1.79)\end{array}$ & $\begin{array}{c}1.70 \\
(1.48)\end{array}$ & $\begin{array}{c}1.82 \\
(1.52)\end{array}$ & $\begin{array}{c}1.76 \\
(1.50)\end{array}$ \\
\hline $\mathbf{P}_{1} \mathbf{K}_{2}$ & $\begin{array}{c}2.46 \\
(1.72)\end{array}$ & $\begin{array}{c}2.51 \\
(1.73)\end{array}$ & $\begin{array}{c}2.49 \\
(1.73)\end{array}$ & $\begin{array}{c}1.51 \\
(1.42)\end{array}$ & $\begin{array}{c}1.55 \\
(1.43)\end{array}$ & $\begin{array}{c}1.53 \\
(1.43)\end{array}$ \\
\hline $\mathbf{P}_{1} \mathbf{K}_{3}$ & $\begin{array}{c}2.27 \\
(1.66)\end{array}$ & $\begin{array}{c}2.22 \\
(1.65)\end{array}$ & $\begin{array}{c}2.25 \\
(1.66)\end{array}$ & $\begin{array}{c}1.39 \\
(1.37)\end{array}$ & $\begin{array}{c}1.40 \\
(1.38)\end{array}$ & $\begin{array}{c}1.40 \\
(1.38)\end{array}$ \\
\hline $\mathbf{P}_{2} \mathbf{K}_{1}$ & $\begin{array}{c}2.65 \\
(1.77)\end{array}$ & $\begin{array}{c}2.77 \\
(1.81)\end{array}$ & $\begin{array}{c}2.78 \\
(1.79)\end{array}$ & $\begin{array}{c}1.74 \\
(1.50)\end{array}$ & $\begin{array}{c}1.88 \\
(1.54)\end{array}$ & $\begin{array}{c}1.81 \\
(1.52)\end{array}$ \\
\hline $\mathbf{P}_{2} \mathbf{K}_{2}$ & $\begin{array}{c}2.49 \\
(1.73)\end{array}$ & $\begin{array}{c}2.53 \\
(1.74)\end{array}$ & $\begin{array}{c}2.53 \\
(1.73)\end{array}$ & $\begin{array}{c}1.54 \\
(1.43)\end{array}$ & $\begin{array}{c}1.60 \\
(1.45)\end{array}$ & $\begin{array}{c}1.57 \\
(1.44)\end{array}$ \\
\hline $\mathbf{P}_{2} \mathbf{K}_{3}$ & $\begin{array}{c}2.32 \\
(1.68)\end{array}$ & $\begin{array}{c}2.35 \\
(1.69) \\
\end{array}$ & $\begin{array}{c}2.44 \\
(1.68)\end{array}$ & $\begin{array}{c}1.38 \\
(1.37) \\
\end{array}$ & $\begin{array}{c}1.44 \\
(1.39)\end{array}$ & $\begin{array}{c}1.40 \\
(1.38) \\
\end{array}$ \\
\hline S.Em. \pm & 0.006 & 0.008 & 0.006 & 0.010 & 0.009 & 0.006 \\
\hline C.D. at 5\% & NS & NS & NS & NS & NS & NS \\
\hline $\mathbf{N}_{1} \mathbf{P}_{1} \mathbf{K}_{1}$ & $\begin{array}{c}2.54 \\
(1.74)\end{array}$ & $\begin{array}{c}2.66 \\
(1.78)\end{array}$ & $\begin{array}{c}2.60 \\
(1.76)\end{array}$ & $\begin{array}{c}1.45 \\
(1.40)\end{array}$ & $\begin{array}{c}1.70 \\
(1.48)\end{array}$ & $\begin{array}{c}1.57 \\
(1.44)\end{array}$ \\
\hline $\mathbf{N}_{1} \mathbf{P}_{1} \mathbf{K}_{2}$ & $\begin{array}{c}2.40 \\
(1.70)\end{array}$ & $\begin{array}{c}2.41 \\
(1.71)\end{array}$ & $\begin{array}{c}2.41 \\
(1.70)\end{array}$ & $\begin{array}{c}1.39 \\
(1.37)\end{array}$ & $\begin{array}{c}1.53 \\
(1.42)\end{array}$ & $\begin{array}{c}1.46 \\
(1.40)\end{array}$ \\
\hline $\mathbf{N}_{1} \mathbf{P}_{1} \mathbf{K}_{3}$ & $\begin{array}{c}2.16 \\
(1.63)\end{array}$ & $\begin{array}{c}2.13 \\
(1.62)\end{array}$ & $\begin{array}{c}2.15 \\
(1.63)\end{array}$ & $\begin{array}{c}1.31 \\
(1.35)\end{array}$ & $\begin{array}{c}1.44 \\
(1.39)\end{array}$ & $\begin{array}{c}1.38 \\
(1.37)\end{array}$ \\
\hline $\mathbf{N}_{1} \mathbf{P}_{2} \mathbf{K}_{1}$ & $\begin{array}{c}2.52 \\
(1.74)\end{array}$ & $\begin{array}{c}2.70 \\
(1.79)\end{array}$ & $\begin{array}{c}2.61 \\
(1.76)\end{array}$ & $\begin{array}{c}1.50 \\
(1.41)\end{array}$ & $\begin{array}{c}1.73 \\
(1.49)\end{array}$ & $\begin{array}{c}1.61 \\
(1.45)\end{array}$ \\
\hline $\mathbf{N}_{1} \mathbf{P}_{2} \mathbf{K}_{2}$ & $\begin{array}{c}2.44 \\
(1.71)\end{array}$ & $\begin{array}{c}2.47 \\
(1.72)\end{array}$ & $\begin{array}{c}2.45 \\
(1.72)\end{array}$ & $\begin{array}{c}1.45 \\
(1.40)\end{array}$ & $\begin{array}{c}1.53 \\
(1.42)\end{array}$ & $\begin{array}{c}1.49 \\
(1.41)\end{array}$ \\
\hline $\mathbf{N}_{1} \mathbf{P}_{2} \mathbf{K}_{3}$ & $\begin{array}{c}2.21 \\
(1.65)\end{array}$ & $\begin{array}{c}2.21 \\
(1.65)\end{array}$ & $\begin{array}{c}2.21 \\
(1.65)\end{array}$ & $\begin{array}{c}1.30 \\
(1.34)\end{array}$ & $\begin{array}{c}1.45 \\
(1.40)\end{array}$ & $\begin{array}{c}1.38 \\
(1.37)\end{array}$ \\
\hline $\mathbf{N}_{2} \mathbf{P}_{1} \mathbf{K}_{1}$ & $\begin{array}{c}2.68 \\
(1.78)\end{array}$ & $\begin{array}{c}2.79 \\
(1.81)\end{array}$ & $\begin{array}{c}2.74 \\
(1.80)\end{array}$ & $\begin{array}{c}1.76 \\
(1.50)\end{array}$ & $\begin{array}{c}1.82 \\
(1.52)\end{array}$ & $\begin{array}{c}1.79 \\
(1.51)\end{array}$ \\
\hline $\mathbf{N}_{2} \mathbf{P}_{1} \mathbf{K}_{2}$ & $\begin{array}{c}2.54 \\
(1.74)\end{array}$ & $\begin{array}{c}2.61 \\
(1.76)\end{array}$ & $\begin{array}{c}2.58 \\
(1.75)\end{array}$ & $\begin{array}{c}1.56 \\
(1.43)\end{array}$ & $\begin{array}{c}1.58 \\
(1.44)\end{array}$ & $\begin{array}{c}1.57 \\
(1.44)\end{array}$ \\
\hline $\mathbf{N}_{2} \mathbf{P}_{1} \mathbf{K}_{3}$ & $\begin{array}{c}2.29 \\
(1.67)\end{array}$ & $\begin{array}{c}2.22 \\
(1.65)\end{array}$ & $\begin{array}{c}2.26 \\
(1.66)\end{array}$ & $\begin{array}{c}1.40 \\
(1.38)\end{array}$ & $\begin{array}{c}1.47 \\
(1.40)\end{array}$ & $\begin{array}{c}1.44 \\
(1.39)\end{array}$ \\
\hline $\mathbf{N}_{2} \mathbf{P}_{2} \mathbf{K}_{1}$ & $\begin{array}{c}2.69 \\
(1.79)\end{array}$ & $\begin{array}{c}2.78 \\
(1.81)\end{array}$ & $\begin{array}{c}2.74 \\
(1.80)\end{array}$ & $\begin{array}{c}1.80 \\
(1.52)\end{array}$ & $\begin{array}{c}1.89 \\
(1.55)\end{array}$ & $\begin{array}{c}1.85 \\
(1.53)\end{array}$ \\
\hline $\mathbf{N}_{2} \mathbf{P}_{2} \mathbf{K}_{2}$ & $\begin{array}{c}2.47 \\
(1.72)\end{array}$ & $\begin{array}{c}2.51 \\
(1.73)\end{array}$ & $\begin{array}{c}2.49 \\
(1.73)\end{array}$ & $\begin{array}{c}1.53 \\
(1.42)\end{array}$ & $\begin{array}{c}1.50 \\
(1.41)\end{array}$ & $\begin{array}{c}1.51 \\
(1.42)\end{array}$ \\
\hline $\mathbf{N}_{2} \mathbf{P}_{2} \mathbf{K}_{3}$ & $\begin{array}{c}2.24 \\
(1.65)\end{array}$ & $\begin{array}{c}2.26 \\
(1.66)\end{array}$ & $\begin{array}{c}2.25 \\
(1.66)\end{array}$ & $\begin{array}{c}1.33 \\
(1.35)\end{array}$ & $\begin{array}{c}1.34 \\
(1.35)\end{array}$ & $\begin{array}{c}1.33 \\
(1.35)\end{array}$ \\
\hline $\mathbf{N}_{3} \mathbf{P}_{1} \mathbf{K}_{1}$ & $\begin{array}{c}2.74 \\
(1.80)\end{array}$ & $\begin{array}{c}2.81 \\
(1.82)\end{array}$ & $\begin{array}{c}2.77 \\
(1.81)\end{array}$ & $\begin{array}{c}1.88 \\
(1.54)\end{array}$ & $\begin{array}{c}1.95 \\
(1.57)\end{array}$ & $\begin{array}{c}1.92 \\
(1.55)\end{array}$ \\
\hline $\mathbf{N}_{3} \mathbf{P}_{1} \mathbf{K}_{2}$ & $\begin{array}{c}2.45 \\
(1.72)\end{array}$ & $\begin{array}{c}2.51 \\
(1.74)\end{array}$ & $\begin{array}{c}2.48 \\
(1.73)\end{array}$ & $\begin{array}{c}1.60 \\
(1.45)\end{array}$ & $\begin{array}{c}1.56 \\
(1.43)\end{array}$ & $\begin{array}{c}1.58 \\
(1.44)\end{array}$ \\
\hline $\mathbf{N}_{3} \mathbf{P}_{1} \mathbf{K}_{3}$ & $\begin{array}{c}2.36 \\
(1.69)\end{array}$ & $\begin{array}{c}2.32 \\
(1.68)\end{array}$ & $\begin{array}{c}2.34 \\
(1.68)\end{array}$ & $\begin{array}{c}1.45 \\
(1.40)\end{array}$ & $\begin{array}{c}1.29 \\
(1.34)\end{array}$ & $\begin{array}{c}1.37 \\
(1.37)\end{array}$ \\
\hline $\mathbf{N}_{3} \mathbf{P}_{2} \mathbf{K}_{1}$ & $\begin{array}{c}2.73 \\
(1.80)\end{array}$ & $\begin{array}{c}2.84 \\
(1.83)\end{array}$ & $\begin{array}{c}2.79 \\
(1.81)\end{array}$ & $\begin{array}{c}1.92 \\
(1.56)\end{array}$ & $\begin{array}{c}2.03 \\
(1.59)\end{array}$ & $\begin{array}{c}1.98 \\
(1.57)\end{array}$ \\
\hline $\mathbf{N}_{3} \mathbf{P}_{2} \mathbf{K}_{2}$ & $\begin{array}{c}2.56 \\
(1.75)\end{array}$ & $\begin{array}{c}2.60 \\
(1.76)\end{array}$ & $\begin{array}{c}2.58 \\
(1.76)\end{array}$ & $\begin{array}{c}1.64 \\
(1.46)\end{array}$ & $\begin{array}{c}1.76 \\
(1.50)\end{array}$ & $\begin{array}{c}1.70 \\
(1.48)\end{array}$ \\
\hline $\mathbf{N}_{3} \mathbf{P}_{2} \mathbf{K}_{3}$ & $\begin{array}{c}2.50 \\
(1.73)\end{array}$ & $\begin{array}{c}2.58 \\
(1.75)\end{array}$ & $\begin{array}{c}2.54 \\
(1.74)\end{array}$ & $\begin{array}{c}1.50 \\
(1.41)\end{array}$ & $\begin{array}{c}1.54 \\
(1.43)\end{array}$ & $\begin{array}{c}1.52 \\
(1.42)\end{array}$ \\
\hline S.Em. \pm & 0.011 & 0.014 & 0.010 & 0.017 & 0.015 & 0.011 \\
\hline C.D. at 5\% & NS & NS & NS & NS & 0.043 & NS \\
\hline Control & $\begin{array}{c}2.93 \\
(1.85)\end{array}$ & $\begin{array}{c}2.97 \\
(1.86)\end{array}$ & $\begin{array}{c}2.95 \\
(1.86)\end{array}$ & $\begin{array}{c}2.35 \\
(1.69)\end{array}$ & $\begin{array}{c}2.94 \\
(1.86)\end{array}$ & $\begin{array}{c}2.65 \\
(1.77)\end{array}$ \\
\hline S.Em. \pm & 0.011 & 0.013 & 0.010 & 0.017 & 0.015 & 0.011 \\
\hline C.D. at 5\% & 0.032 & 0.038 & 0.028 & 0.048 & 0.042 & 0.032 \\
\hline
\end{tabular}


Table. 2 Jassids population (per 3 leaves) in Bt cotton as influenced by different levels of NPK in Alfisol

\begin{tabular}{|c|c|c|c|c|c|c|}
\hline \multirow[t]{3}{*}{ Treatments } & \multicolumn{6}{|c|}{ Jassids (per 3 leaves) } \\
\hline & \multicolumn{3}{|c|}{70 DAS } & \multicolumn{3}{|c|}{90 DAS } \\
\hline & 2012-13 & 2013-14 & Pooled & 2012-13 & 2013-14 & Pooled \\
\hline $\mathbf{N}_{1}$ & $\begin{array}{c}2.15 \\
(1.63)\end{array}$ & $\begin{array}{c}1.98 \\
(1.57)\end{array}$ & $\begin{array}{c}2.07 \\
(1.60)\end{array}$ & $\begin{array}{c}1.20 \\
(1.30) \\
\end{array}$ & $\begin{array}{c}1.26 \\
(1.33)\end{array}$ & $\begin{array}{c}1.23 \\
(1.31)\end{array}$ \\
\hline $\mathbf{N}_{2}$ & $\begin{array}{c}2.15 \\
(1.63)\end{array}$ & $\begin{array}{c}2.04 \\
(1.59)\end{array}$ & $\begin{array}{c}2.10 \\
(1.61)\end{array}$ & $\begin{array}{c}1.31 \\
(1.34)\end{array}$ & $\begin{array}{c}1.48 \\
(1.40)\end{array}$ & $\begin{array}{c}1.39 \\
(1.37)\end{array}$ \\
\hline $\mathbf{N}_{3}$ & $\begin{array}{c}2.19 \\
(1.64)\end{array}$ & $\begin{array}{c}2.09 \\
(1.61)\end{array}$ & $\begin{array}{c}2.14 \\
(1.62)\end{array}$ & $\begin{array}{c}1.44 \\
(1.39)\end{array}$ & $\begin{array}{c}1.66 \\
(1.47)\end{array}$ & $\begin{array}{c}1.55 \\
(1.43)\end{array}$ \\
\hline S.Em. \pm & 0.005 & 0.005 & 0.004 & 0.006 & 0.003 & 0.003 \\
\hline C.D. at $5 \%$ & NS & 0.015 & 0.011 & 0.018 & 0.010 & 0.010 \\
\hline $\mathbf{P}_{1}$ & $\begin{array}{c}2.15 \\
(1.63)\end{array}$ & $\begin{array}{c}2.02 \\
(1.59)\end{array}$ & $\begin{array}{c}2.09 \\
(1.61)\end{array}$ & $\begin{array}{c}1.30 \\
(1.34)\end{array}$ & $\begin{array}{c}1.44 \\
(1.39)\end{array}$ & $\begin{array}{c}1.37 \\
(1.36)\end{array}$ \\
\hline $\mathbf{P}_{2}$ & $\begin{array}{c}2.18 \\
(1.64)\end{array}$ & $\begin{array}{c}2.05 \\
(1.60)\end{array}$ & $\begin{array}{c}2.12 \\
(1.62)\end{array}$ & $\begin{array}{c}1.33 \\
(1.35)\end{array}$ & $\begin{array}{c}1.49 \\
(1.41)\end{array}$ & $\begin{array}{c}1.41 \\
(1.38)\end{array}$ \\
\hline S.Em. \pm & 0.004 & 0.004 & 0.003 & 0.005 & 0.003 & 0.003 \\
\hline C.D. at $5 \%$ & NS & NS & NS & NS & 0.008 & 0.008 \\
\hline $\mathbf{K}_{1}$ & $\begin{array}{c}2.36 \\
(1.69)\end{array}$ & $\begin{array}{c}2.29 \\
(1.67)\end{array}$ & $\begin{array}{c}2.32 \\
(1.68)\end{array}$ & $\begin{array}{c}1.52 \\
(1.42)\end{array}$ & $\begin{array}{c}1.61 \\
(1.45)\end{array}$ & $\begin{array}{c}1.56 \\
(1.43)\end{array}$ \\
\hline $\mathbf{K}_{2}$ & $\begin{array}{c}2.17 \\
(1.63)\end{array}$ & $\begin{array}{c}2.03 \\
(1.59)\end{array}$ & $\begin{array}{c}2.10 \\
(1.61)\end{array}$ & $\begin{array}{c}1.29 \\
(1.34)\end{array}$ & $\begin{array}{c}1.47 \\
(1.40)\end{array}$ & $\begin{array}{c}1.38 \\
(1.37)\end{array}$ \\
\hline $\mathbf{K}_{\mathbf{3}}$ & $\begin{array}{c}1.98 \\
(1.57)\end{array}$ & $\begin{array}{c}1.79 \\
(1.51)\end{array}$ & $\begin{array}{c}1.88 \\
(1.54)\end{array}$ & $\begin{array}{c}1.14 \\
(1.28)\end{array}$ & $\begin{array}{c}1.32 \\
(1.35)\end{array}$ & $\begin{array}{c}1.23 \\
(1.31)\end{array}$ \\
\hline S.Em. \pm & 0.005 & 0.005 & 0.004 & 0.006 & 0.003 & 0.003 \\
\hline C.D. at 5\% & 0.015 & 0.015 & 0.011 & 0.018 & 0.010 & 0.010 \\
\hline $\mathbf{N}_{1} \mathbf{P}_{1}$ & $\begin{array}{c}2.14 \\
(1.63)\end{array}$ & $\begin{array}{c}1.96 \\
(1.57)\end{array}$ & $\begin{array}{c}2.05 \\
(1.60)\end{array}$ & $\begin{array}{c}1.17 \\
(1.29)\end{array}$ & $\begin{array}{c}1.24 \\
(1.32)\end{array}$ & $\begin{array}{c}1.21 \\
(1.31)\end{array}$ \\
\hline $\mathbf{N}_{1} \mathbf{P}_{2}$ & $\begin{array}{c}2.16 \\
(1.63)\end{array}$ & $\begin{array}{c}2.00 \\
(1.58)\end{array}$ & $\begin{array}{c}2.08 \\
(1.61)\end{array}$ & $\begin{array}{c}1.22 \\
(1.31)\end{array}$ & $\begin{array}{c}1.28 \\
(1.33)\end{array}$ & $\begin{array}{c}1.25 \\
(1.32)\end{array}$ \\
\hline $\mathbf{N}_{2} \mathbf{P}_{1}$ & $\begin{array}{c}2.15 \\
(1.63)\end{array}$ & $\begin{array}{c}2.02 \\
(1.58)\end{array}$ & $\begin{array}{c}2.09 \\
(1.61)\end{array}$ & $\begin{array}{c}1.30 \\
(1.34)\end{array}$ & $\begin{array}{c}1.43 \\
(1.39)\end{array}$ & $\begin{array}{c}1.37 \\
(1.36)\end{array}$ \\
\hline $\mathbf{N}_{2} \mathbf{P}_{2}$ & $\begin{array}{c}2.15 \\
(1.63)\end{array}$ & $\begin{array}{c}2.06 \\
(1.60)\end{array}$ & $\begin{array}{c}2.11 \\
(1.61)\end{array}$ & $\begin{array}{c}1.32 \\
(1.35)\end{array}$ & $\begin{array}{c}1.52 \\
(1.42)\end{array}$ & $\begin{array}{c}1.42 \\
(1.38)\end{array}$ \\
\hline $\mathbf{N}_{3} \mathbf{P}_{1}$ & $\begin{array}{c}2.15 \\
(1.63)\end{array}$ & $\begin{array}{c}2.10 \\
(1.61)\end{array}$ & $\begin{array}{c}2.12 \\
(1.62)\end{array}$ & $\begin{array}{c}1.43 \\
(1.39)\end{array}$ & $\begin{array}{c}1.64 \\
(1.46)\end{array}$ & $\begin{array}{c}1.53 \\
(1.42)\end{array}$ \\
\hline $\mathbf{N}_{3} \mathbf{P}_{2}$ & $\begin{array}{c}2.24 \\
(1.65)\end{array}$ & $\begin{array}{c}2.09 \\
(1.61)\end{array}$ & $\begin{array}{c}2.16 \\
(1.63)\end{array}$ & $\begin{array}{c}1.45 \\
(1.39)\end{array}$ & $\begin{array}{c}1.68 \\
(1.48)\end{array}$ & $\begin{array}{c}1.56 \\
(1.43)\end{array}$ \\
\hline S.Em. \pm & 0.007 & 0.008 & 0.006 & 0.009 & 0.005 & 0.005 \\
\hline C.D. at $5 \%$ & NS & NS & NS & NS & NS & NS \\
\hline $\mathbf{N}_{1} \mathrm{~K}_{1}$ & $\begin{array}{c}2.24 \\
(1.65)\end{array}$ & $\begin{array}{c}2.09 \\
(1.61)\end{array}$ & $\begin{array}{c}2.16 \\
(1.63)\end{array}$ & $\begin{array}{c}1.27 \\
(1.33)\end{array}$ & $\begin{array}{c}1.36 \\
(1.36)\end{array}$ & $\begin{array}{c}1.31 \\
(1.35)\end{array}$ \\
\hline $\mathbf{N}_{1} \mathbf{K}_{2}$ & $\begin{array}{c}2.14 \\
(1.63)\end{array}$ & $\begin{array}{c}2.01 \\
(1.58)\end{array}$ & $\begin{array}{c}2.08 \\
(1.61)\end{array}$ & $\begin{array}{c}1.20 \\
(1.30)\end{array}$ & $\begin{array}{c}1.25 \\
(1.32)\end{array}$ & $\begin{array}{c}1.23 \\
(1.31)\end{array}$ \\
\hline $\mathbf{N}_{1} \mathbf{K}_{3}$ & $\begin{array}{c}2.07 \\
(1.60)\end{array}$ & $\begin{array}{c}1.84 \\
(1.53)\end{array}$ & $\begin{array}{c}1.96 \\
(1.57)\end{array}$ & $\begin{array}{c}1.12 \\
(1.27)\end{array}$ & $\begin{array}{c}1.17 \\
(1.29)\end{array}$ & $\begin{array}{c}1.15 \\
(1.28)\end{array}$ \\
\hline $\mathbf{N}_{2} \mathbf{K}_{1}$ & $\begin{array}{c}2.36 \\
(1.69)\end{array}$ & $\begin{array}{c}2.37 \\
(1.69)\end{array}$ & $\begin{array}{c}2.36 \\
(1.69)\end{array}$ & $\begin{array}{c}1.57 \\
(1.44)\end{array}$ & $\begin{array}{c}1.63 \\
(1.46)\end{array}$ & $\begin{array}{c}1.60 \\
(1.45)\end{array}$ \\
\hline $\mathbf{N}_{2} \mathbf{K}_{2}$ & $\begin{array}{c}2.19 \\
(1.64)\end{array}$ & $\begin{array}{c}2.01 \\
(1.58)\end{array}$ & $\begin{array}{c}2.10 \\
(1.61)\end{array}$ & $\begin{array}{c}1.26 \\
(1.33)\end{array}$ & $\begin{array}{c}1.50 \\
(1.41)\end{array}$ & $\begin{array}{c}1.38 \\
(1.37)\end{array}$ \\
\hline $\mathbf{N}_{2} \mathbf{K}_{3}$ & $\begin{array}{c}1.91 \\
(1.55)\end{array}$ & $\begin{array}{c}1.73 \\
(1.49)\end{array}$ & $\begin{array}{c}1.82 \\
(1.52)\end{array}$ & $\begin{array}{c}1.10 \\
(1.27)\end{array}$ & $\begin{array}{c}1.30 \\
(1.34)\end{array}$ & $\begin{array}{c}1.20 \\
(1.30)\end{array}$ \\
\hline $\mathbf{N}_{3} \mathbf{K}_{1}$ & $\begin{array}{c}2.47 \\
(1.72)\end{array}$ & $\begin{array}{c}2.40 \\
(1.70)\end{array}$ & $\begin{array}{c}2.44 \\
(1.71)\end{array}$ & $\begin{array}{c}1.73 \\
(1.49)\end{array}$ & $\begin{array}{c}1.83 \\
(1.53)\end{array}$ & $\begin{array}{c}1.78 \\
(1.51)\end{array}$ \\
\hline $\mathbf{N}_{3} \mathbf{K}_{2}$ & $\begin{array}{c}2.16 \\
(1.63)\end{array}$ & $\begin{array}{c}2.09 \\
(1.61)\end{array}$ & $\begin{array}{c}2.12 \\
(1.62)\end{array}$ & $\begin{array}{c}1.40 \\
(1.38)\end{array}$ & $\begin{array}{c}1.67 \\
(1.47)\end{array}$ & $\begin{array}{c}1.53 \\
(1.42)\end{array}$ \\
\hline $\mathbf{N}_{3} \mathbf{K}_{3}$ & $\begin{array}{c}1.95 \\
(1.56)\end{array}$ & $\begin{array}{c}1.79 \\
(1.51)\end{array}$ & $\begin{array}{c}1.87 \\
(1.54)\end{array}$ & $\begin{array}{c}1.20 \\
(1.30)\end{array}$ & $\begin{array}{c}1.48 \\
(1.41)\end{array}$ & $\begin{array}{c}1.34 \\
(1.35)\end{array}$ \\
\hline S.Em. \pm & 0.009 & 0.009 & 0.007 & 0.011 & 0.006 & 0.006 \\
\hline C.D. at 5\% & 0.026 & 0.027 & 0.020 & 0.032 & 0.017 & 0.017 \\
\hline
\end{tabular}


Contd.

\begin{tabular}{|c|c|c|c|c|c|c|}
\hline \multirow[t]{3}{*}{ Treatments } & \multicolumn{6}{|c|}{ Jassids (per 3 leaves) } \\
\hline & \multicolumn{3}{|c|}{70 DAS } & \multicolumn{3}{|c|}{90 DAS } \\
\hline & 2012-13 & 2013-14 & Pooled & 2012-13 & 2013-14 & Pooled \\
\hline $\mathbf{P}_{1} \mathbf{K}_{1}$ & $\begin{array}{c}2.34 \\
(1.68)\end{array}$ & $\begin{array}{c}2.27 \\
(1.66)\end{array}$ & $\begin{array}{c}2.30 \\
(1.67)\end{array}$ & $\begin{array}{c}1.48 \\
(1.41)\end{array}$ & $\begin{array}{c}1.59 \\
(1.44)\end{array}$ & $\begin{array}{c}1.54 \\
(1.43)\end{array}$ \\
\hline $\mathbf{P}_{1} \mathbf{K}_{2}$ & $\begin{array}{c}2.14 \\
(1.62)\end{array}$ & $\begin{array}{c}2.01 \\
(1.59)\end{array}$ & $\begin{array}{c}2.08 \\
(1.61)\end{array}$ & $\begin{array}{c}1.30 \\
(1.34)\end{array}$ & $\begin{array}{c}1.44 \\
(1.39)\end{array}$ & $\begin{array}{c}1.37 \\
(1.37)\end{array}$ \\
\hline $\mathbf{P}_{1} \mathbf{K}_{3}$ & $\begin{array}{c}1.97 \\
(1.57)\end{array}$ & $\begin{array}{c}1.78 \\
(1.51)\end{array}$ & $\begin{array}{c}1.88 \\
(1.54)\end{array}$ & $\begin{array}{c}1.12 \\
(1.27)\end{array}$ & $\begin{array}{c}1.28 \\
(1.33)\end{array}$ & $\begin{array}{c}1.20 \\
(1.30)\end{array}$ \\
\hline $\mathbf{P}_{2} \mathbf{K}_{1}$ & $\begin{array}{c}2.37 \\
(1.70)\end{array}$ & $\begin{array}{c}2.30 \\
(1.67)\end{array}$ & $\begin{array}{c}2.34 \\
(1.68)\end{array}$ & $\begin{array}{c}1.56 \\
(1.43)\end{array}$ & $\begin{array}{c}1.62 \\
(1.45)\end{array}$ & $\begin{array}{c}1.59 \\
(1.44)\end{array}$ \\
\hline $\mathbf{P}_{2} \mathbf{K}_{2}$ & $\begin{array}{c}2.19 \\
(1.64)\end{array}$ & $\begin{array}{c}2.05 \\
(1.60)\end{array}$ & $\begin{array}{c}2.12 \\
(1.62)\end{array}$ & $\begin{array}{c}1.27 \\
(1.33)\end{array}$ & $\begin{array}{c}1.50 \\
(1.41)\end{array}$ & $\begin{array}{c}1.39 \\
(1.37)\end{array}$ \\
\hline $\mathbf{P}_{2} \mathbf{K}_{3}$ & $\begin{array}{c}1.98 \\
(1.57)\end{array}$ & $\begin{array}{c}1.79 \\
(1.51) \\
\end{array}$ & $\begin{array}{c}1.89 \\
(1.54)\end{array}$ & $\begin{array}{c}1.16 \\
(1.29) \\
\end{array}$ & $\begin{array}{c}1.35 \\
(1.36)\end{array}$ & $\begin{array}{c}1.26 \\
(1.32) \\
\end{array}$ \\
\hline S.Em. \pm & 0.007 & 0.008 & 0.006 & 0.009 & 0.005 & 0.005 \\
\hline C.D. at 5\% & NS & NS & NS & NS & NS & NS \\
\hline $\mathbf{N}_{1} \mathbf{P}_{1} \mathbf{K}_{1}$ & $\begin{array}{c}2.21 \\
(1.65)\end{array}$ & $\begin{array}{c}2.09 \\
(1.61)\end{array}$ & $\begin{array}{c}2.15 \\
(1.63)\end{array}$ & $\begin{array}{c}1.23 \\
(1.32)\end{array}$ & $\begin{array}{c}1.35 \\
(1.36)\end{array}$ & $\begin{array}{c}1.29 \\
(1.34)\end{array}$ \\
\hline $\mathbf{N}_{1} \mathbf{P}_{1} \mathbf{K}_{2}$ & $\begin{array}{c}2.14 \\
(1.62)\end{array}$ & $\begin{array}{c}1.99 \\
(1.58)\end{array}$ & $\begin{array}{c}2.06 \\
(1.60)\end{array}$ & $\begin{array}{c}1.18 \\
(1.30)\end{array}$ & $\begin{array}{c}1.24 \\
(1.32)\end{array}$ & $\begin{array}{c}1.21 \\
(1.31)\end{array}$ \\
\hline $\mathbf{N}_{1} \mathbf{P}_{1} \mathbf{K}_{3}$ & $\begin{array}{c}2.09 \\
(1.61)\end{array}$ & $\begin{array}{c}1.79 \\
(1.51)\end{array}$ & $\begin{array}{c}1.94 \\
(1.56)\end{array}$ & $\begin{array}{c}1.09 \\
(1.26)\end{array}$ & $\begin{array}{c}1.15 \\
(1.28)\end{array}$ & $\begin{array}{c}1.12 \\
(1.27)\end{array}$ \\
\hline $\mathbf{N}_{1} \mathbf{P}_{2} \mathbf{K}_{1}$ & $\begin{array}{c}2.27 \\
(1.66)\end{array}$ & $\begin{array}{c}2.08 \\
(1.61)\end{array}$ & $\begin{array}{c}2.18 \\
(1.64)\end{array}$ & $\begin{array}{c}1.30 \\
(1.34)\end{array}$ & $\begin{array}{c}1.37 \\
(1.37)\end{array}$ & $\begin{array}{c}1.34 \\
(1.35)\end{array}$ \\
\hline $\mathbf{N}_{1} \mathbf{P}_{2} \mathbf{K}_{2}$ & $\begin{array}{c}2.14 \\
(1.63)\end{array}$ & $\begin{array}{c}2.04 \\
(1.59)\end{array}$ & $\begin{array}{c}2.09 \\
(1.61)\end{array}$ & $\begin{array}{c}1.21 \\
(1.31)\end{array}$ & $\begin{array}{c}1.27 \\
(1.33)\end{array}$ & $\begin{array}{c}1.24 \\
(1.32)\end{array}$ \\
\hline $\mathbf{N}_{1} \mathbf{P}_{2} \mathbf{K}_{3}$ & $\begin{array}{c}2.06 \\
(1.60)\end{array}$ & $\begin{array}{c}1.89 \\
(1.55)\end{array}$ & $\begin{array}{c}1.98 \\
(1.57)\end{array}$ & $\begin{array}{c}1.15 \\
(1.28)\end{array}$ & $\begin{array}{c}1.20 \\
(1.30)\end{array}$ & $\begin{array}{c}1.17 \\
(1.29)\end{array}$ \\
\hline $\mathbf{N}_{2} \mathbf{P}_{1} \mathbf{K}_{1}$ & $\begin{array}{c}2.34 \\
(1.68)\end{array}$ & $\begin{array}{c}2.33 \\
(1.68)\end{array}$ & $\begin{array}{c}2.33 \\
(1.68)\end{array}$ & $\begin{array}{c}1.54 \\
(1.43)\end{array}$ & $\begin{array}{c}1.61 \\
(1.45)\end{array}$ & $\begin{array}{c}1.58 \\
(1.44)\end{array}$ \\
\hline $\mathbf{N}_{2} \mathbf{P}_{1} \mathbf{K}_{2}$ & $\begin{array}{c}2.18 \\
(1.64)\end{array}$ & $\begin{array}{c}1.93 \\
(1.56)\end{array}$ & $\begin{array}{c}2.06 \\
(1.60)\end{array}$ & $\begin{array}{c}1.27 \\
(1.33)\end{array}$ & $\begin{array}{c}1.41 \\
(1.38)\end{array}$ & $\begin{array}{c}1.34 \\
(1.36)\end{array}$ \\
\hline $\mathbf{N}_{2} \mathbf{P}_{1} \mathbf{K}_{3}$ & $\begin{array}{c}1.95 \\
(1.56)\end{array}$ & $\begin{array}{c}1.79 \\
(1.51)\end{array}$ & $\begin{array}{c}1.87 \\
(1.54)\end{array}$ & $\begin{array}{c}1.08 \\
(1.26)\end{array}$ & $\begin{array}{c}1.28 \\
(1.33)\end{array}$ & $\begin{array}{c}1.18 \\
(1.30)\end{array}$ \\
\hline $\mathbf{N}_{2} \mathbf{P}_{2} \mathbf{K}_{1}$ & $\begin{array}{c}2.38 \\
(1.70)\end{array}$ & $\begin{array}{c}2.41 \\
(1.71)\end{array}$ & $\begin{array}{c}2.40 \\
(1.70)\end{array}$ & $\begin{array}{c}1.59 \\
(1.45)\end{array}$ & $\begin{array}{c}1.65 \\
(1.47)\end{array}$ & $\begin{array}{c}1.62 \\
(1.46)\end{array}$ \\
\hline $\mathbf{N}_{2} \mathbf{P}_{2} \mathbf{K}_{2}$ & $\begin{array}{c}2.20 \\
(1.64)\end{array}$ & $\begin{array}{c}2.08 \\
(1.61)\end{array}$ & $\begin{array}{c}2.14 \\
(1.63)\end{array}$ & $\begin{array}{c}1.25 \\
(1.32)\end{array}$ & $\begin{array}{c}1.59 \\
(1.45)\end{array}$ & $\begin{array}{c}1.42 \\
(1.38)\end{array}$ \\
\hline $\mathbf{N}_{2} \mathbf{P}_{2} \mathbf{K}_{3}$ & $\begin{array}{c}1.87 \\
(1.54)\end{array}$ & $\begin{array}{c}1.68 \\
(1.48)\end{array}$ & $\begin{array}{c}1.78 \\
(1.51)\end{array}$ & $\begin{array}{c}1.12 \\
(1.27)\end{array}$ & $\begin{array}{c}1.32 \\
(1.35)\end{array}$ & $\begin{array}{c}1.22 \\
(1.31)\end{array}$ \\
\hline $\mathbf{N}_{3} \mathbf{P}_{1} \mathbf{K}_{1}$ & $\begin{array}{c}2.47 \\
(1.72)\end{array}$ & $\begin{array}{c}2.39 \\
(1.70)\end{array}$ & $\begin{array}{c}2.43 \\
(1.71)\end{array}$ & $\begin{array}{c}1.67 \\
(1.47)\end{array}$ & $\begin{array}{c}1.81 \\
(1.52)\end{array}$ & $\begin{array}{c}1.74 \\
(1.50)\end{array}$ \\
\hline $\mathbf{N}_{3} \mathbf{P}_{1} \mathbf{K}_{2}$ & $\begin{array}{c}2.10 \\
(1.61)\end{array}$ & $\begin{array}{c}2.13 \\
(1.62)\end{array}$ & $\begin{array}{c}2.12 \\
(1.62)\end{array}$ & $\begin{array}{c}1.44 \\
(1.39)\end{array}$ & $\begin{array}{c}1.69 \\
(1.48)\end{array}$ & $\begin{array}{c}1.56 \\
(1.44)\end{array}$ \\
\hline $\mathbf{N}_{3} \mathbf{P}_{1} \mathbf{K}_{3}$ & $\begin{array}{c}1.88 \\
(1.54)\end{array}$ & $\begin{array}{c}1.77 \\
(1.51)\end{array}$ & $\begin{array}{c}1.83 \\
(1.52)\end{array}$ & $\begin{array}{c}1.18 \\
(1.30)\end{array}$ & $\begin{array}{c}1.42 \\
(1.39)\end{array}$ & $\begin{array}{c}1.30 \\
(1.34)\end{array}$ \\
\hline $\mathbf{N}_{3} \mathbf{P}_{2} \mathbf{K}_{1}$ & $\begin{array}{c}2.48 \\
(1.73)\end{array}$ & $\begin{array}{c}2.42 \\
(1.71)\end{array}$ & $\begin{array}{c}2.45 \\
(1.72)\end{array}$ & $\begin{array}{c}1.78 \\
(1.51)\end{array}$ & $\begin{array}{c}1.85 \\
(1.53)\end{array}$ & $\begin{array}{c}1.81 \\
(1.52)\end{array}$ \\
\hline $\mathbf{N}_{3} \mathbf{P}_{2} \mathbf{K}_{2}$ & $\begin{array}{c}2.22 \\
(1.65)\end{array}$ & $\begin{array}{c}2.04 \\
(1.59)\end{array}$ & $\begin{array}{c}2.13 \\
(1.62)\end{array}$ & $\begin{array}{c}1.35 \\
(1.36)\end{array}$ & $\begin{array}{c}1.65 \\
(1.47)\end{array}$ & $\begin{array}{c}1.50 \\
(1.41)\end{array}$ \\
\hline $\mathbf{N}_{3} \mathbf{P}_{2} \mathbf{K}_{3}$ & $\begin{array}{c}2.01 \\
(1.59)\end{array}$ & $\begin{array}{c}1.80 \\
(1.52)\end{array}$ & $\begin{array}{c}1.91 \\
(1.55)\end{array}$ & $\begin{array}{c}1.21 \\
(1.31)\end{array}$ & $\begin{array}{c}1.54 \\
(1.43)\end{array}$ & $\begin{array}{c}1.37 \\
(1.37)\end{array}$ \\
\hline S.Em. \pm & 0.013 & 0.013 & 0.010 & 0.016 & 0.008 & 0.008 \\
\hline C.D. at 5\% & NS & 0.038 & NS & NS & NS & NS \\
\hline Control & $\begin{array}{c}2.65 \\
(1.78)\end{array}$ & $\begin{array}{c}2.68 \\
(1.78)\end{array}$ & $\begin{array}{c}2.67 \\
(1.78)\end{array}$ & $\begin{array}{c}2.07 \\
(1.60)\end{array}$ & $\begin{array}{c}2.14 \\
(1.62)\end{array}$ & $\begin{array}{c}2.10 \\
(1.61)\end{array}$ \\
\hline S.Em. \pm & 0.012 & 0.013 & 0.010 & 0.015 & 0.008 & 0.008 \\
\hline C.D. at $5 \%$ & 0.036 & 0.037 & 0.027 & 0.044 & 0.024 & 0.024 \\
\hline
\end{tabular}


Table.3 Aphids population (per 3 leaves) in Bt cotton as influenced by different levels of NPK in Alfisol

\begin{tabular}{|c|c|c|c|c|c|c|}
\hline \multirow[t]{3}{*}{ Treatments } & \multicolumn{6}{|c|}{ Aphids (per 3 leaves) } \\
\hline & \multicolumn{3}{|c|}{70 DAS } & \multicolumn{3}{|c|}{90 DAS } \\
\hline & 2012-13 & 2013-14 & Pooled & 2012-13 & 2013-14 & Pooled \\
\hline $\mathbf{N}_{1}$ & $\begin{array}{c}3.12 \\
(1.90)\end{array}$ & $\begin{array}{c}3.48 \\
(1.99)\end{array}$ & $\begin{array}{c}3.30 \\
(1.95)\end{array}$ & $\begin{array}{c}2.17 \\
(1.63)\end{array}$ & $\begin{array}{c}2.02 \\
(1.59)\end{array}$ & $\begin{array}{c}2.09 \\
(1.61)\end{array}$ \\
\hline $\mathbf{N}_{2}$ & $\begin{array}{c}3.04 \\
(1.87)\end{array}$ & $\begin{array}{c}3.67 \\
(2.04)\end{array}$ & $\begin{array}{c}3.35 \\
(1.96)\end{array}$ & $\begin{array}{c}2.27 \\
(1.66)\end{array}$ & $\begin{array}{c}2.25 \\
(1.66)\end{array}$ & $\begin{array}{c}2.26 \\
(1.66)\end{array}$ \\
\hline $\mathbf{N}_{3}$ & $\begin{array}{c}3.20 \\
(1.92)\end{array}$ & $\begin{array}{c}3.77 \\
(2.07)\end{array}$ & $\begin{array}{c}3.49 \\
(1.99)\end{array}$ & $\begin{array}{c}2.40 \\
(1.70)\end{array}$ & $\begin{array}{c}2.35 \\
(1.69)\end{array}$ & $\begin{array}{c}2.37 \\
(1.69)\end{array}$ \\
\hline S.Em. \pm & 0.013 & 0.006 & 0.008 & 0.004 & 0.004 & 0.003 \\
\hline C.D. at $5 \%$ & NS & 0.016 & 0.023 & 0.013 & 0.011 & 0.008 \\
\hline $\mathbf{P}_{1}$ & $\begin{array}{c}3.12 \\
(1.90)\end{array}$ & $\begin{array}{c}3.60 \\
(2.02)\end{array}$ & $\begin{array}{c}3.36 \\
(1.96)\end{array}$ & $\begin{array}{c}2.25 \\
(1.66)\end{array}$ & $\begin{array}{c}2.19 \\
(1.64)\end{array}$ & $\begin{array}{c}2.22 \\
(1.65)\end{array}$ \\
\hline $\mathbf{P}_{2}$ & $\begin{array}{c}3.12 \\
(1.90)\end{array}$ & $\begin{array}{c}3.68 \\
(2.04)\end{array}$ & $\begin{array}{c}3.40 \\
(1.97)\end{array}$ & $\begin{array}{c}2.31 \\
(1.67)\end{array}$ & $\begin{array}{c}2.23 \\
(1.65)\end{array}$ & $\begin{array}{c}2.27 \\
(1.66)\end{array}$ \\
\hline S.Em. \pm & 0.011 & 0.005 & 0.006 & 0.004 & 0.003 & 0.002 \\
\hline C.D. at 5\% & NS & 0.013 & NS & 0.010 & 0.009 & 0.006 \\
\hline $\mathbf{K}_{1}$ & $\begin{array}{c}3.65 \\
(2.04)\end{array}$ & $\begin{array}{c}3.85 \\
(2.09)\end{array}$ & $\begin{array}{c}3.75 \\
(2.06)\end{array}$ & $\begin{array}{c}2.48 \\
(1.72)\end{array}$ & $\begin{array}{c}2.36 \\
(1.69)\end{array}$ & $\begin{array}{c}2.42 \\
(1.71)\end{array}$ \\
\hline $\mathbf{K}_{2}$ & $\begin{array}{c}3.10 \\
(1.90)\end{array}$ & $\begin{array}{c}3.62 \\
(2.03)\end{array}$ & $\begin{array}{c}3.36 \\
(1.96)\end{array}$ & $\begin{array}{c}2.29 \\
(1.67)\end{array}$ & $\begin{array}{c}2.24 \\
(1.65)\end{array}$ & $\begin{array}{c}2.26 \\
(1.66)\end{array}$ \\
\hline $\mathbf{K}_{\mathbf{3}}$ & $\begin{array}{c}2.61 \\
(1.76)\end{array}$ & $\begin{array}{c}3.45 \\
(1.99)\end{array}$ & $\begin{array}{c}3.03 \\
(1.87)\end{array}$ & $\begin{array}{c}2.07 \\
(1.60)\end{array}$ & $\begin{array}{c}2.03 \\
(1.59)\end{array}$ & $\begin{array}{c}2.05 \\
(1.60)\end{array}$ \\
\hline S.Em. \pm & 0.013 & 0.006 & 0.008 & 0.004 & 0.004 & 0.003 \\
\hline C.D. at 5\% & 0.038 & 0.016 & 0.023 & 0.013 & 0.011 & 0.008 \\
\hline $\mathbf{N}_{1} \mathbf{P}_{1}$ & $\begin{array}{c}3.09 \\
(1.89)\end{array}$ & $\begin{array}{c}3.41 \\
(1.98)\end{array}$ & $\begin{array}{c}3.25 \\
(1.93)\end{array}$ & $\begin{array}{c}2.19 \\
(1.64)\end{array}$ & $\begin{array}{c}2.03 \\
(1.59)\end{array}$ & $\begin{array}{c}2.11 \\
(1.61)\end{array}$ \\
\hline $\mathbf{N}_{1} \mathbf{P}_{2}$ & $\begin{array}{c}3.15 \\
(1.91)\end{array}$ & $\begin{array}{c}3.55 \\
(2.01)\end{array}$ & $\begin{array}{c}3.35 \\
(1.96)\end{array}$ & $\begin{array}{c}2.15 \\
(1.63)\end{array}$ & $\begin{array}{c}2.01 \\
(1.58)\end{array}$ & $\begin{array}{c}2.08 \\
(1.61)\end{array}$ \\
\hline $\mathbf{N}_{2} \mathbf{P}_{1}$ & $\begin{array}{c}3.22 \\
(1.93)\end{array}$ & $\begin{array}{c}3.63 \\
(2.03)\end{array}$ & $\begin{array}{c}3.42 \\
(1.98)\end{array}$ & $\begin{array}{c}2.21 \\
(1.64)\end{array}$ & $\begin{array}{c}2.25 \\
(1.66)\end{array}$ & $\begin{array}{c}2.23 \\
(1.65)\end{array}$ \\
\hline $\mathbf{N}_{2} \mathbf{P}_{2}$ & $\begin{array}{c}2.86 \\
(1.82)\end{array}$ & $\begin{array}{c}3.72 \\
(2.05)\end{array}$ & $\begin{array}{c}3.29 \\
(1.94)\end{array}$ & $\begin{array}{c}2.33 \\
(1.68)\end{array}$ & $\begin{array}{c}2.26 \\
(1.66)\end{array}$ & $\begin{array}{c}2.29 \\
(1.67)\end{array}$ \\
\hline $\mathbf{N}_{3} \mathbf{P}_{1}$ & $\begin{array}{c}3.06 \\
(1.88)\end{array}$ & $\begin{array}{c}3.77 \\
(2.07)\end{array}$ & $\begin{array}{c}3.42 \\
(1.97)\end{array}$ & $\begin{array}{c}2.35 \\
(1.69)\end{array}$ & $\begin{array}{c}2.28 \\
(1.67)\end{array}$ & $\begin{array}{c}2.31 \\
(1.68)\end{array}$ \\
\hline $\mathbf{N}_{3} \mathbf{P}_{2}$ & $\begin{array}{c}3.34 \\
(1.95)\end{array}$ & $\begin{array}{c}3.77 \\
(2.07)\end{array}$ & $\begin{array}{c}3.56 \\
(2.01)\end{array}$ & $\begin{array}{c}2.45 \\
(1.72)\end{array}$ & $\begin{array}{c}2.42 \\
(1.71)\end{array}$ & $\begin{array}{c}2.44 \\
(1.71)\end{array}$ \\
\hline S.Em. \pm & 0.019 & 0.008 & 0.011 & 0.006 & 0.006 & 0.004 \\
\hline C.D. at 5\% & 0.054 & NS & 0.032 & 0.018 & 0.016 & 0.011 \\
\hline $\mathbf{N}_{1} \mathbf{K}_{1}$ & $\begin{array}{c}3.37 \\
(1.97)\end{array}$ & $\begin{array}{c}3.59 \\
(2.02)\end{array}$ & $\begin{array}{c}3.48 \\
(1.99)\end{array}$ & $\begin{array}{c}2.30 \\
(1.67)\end{array}$ & $\begin{array}{c}2.17 \\
(1.63)\end{array}$ & $\begin{array}{c}2.24 \\
(1.65)\end{array}$ \\
\hline $\mathbf{N}_{1} \mathbf{K}_{2}$ & $\begin{array}{c}3.13 \\
(1.90)\end{array}$ & $\begin{array}{c}3.50 \\
(2.00)\end{array}$ & $\begin{array}{c}3.31 \\
(1.95)\end{array}$ & $\begin{array}{c}2.19 \\
(1.64)\end{array}$ & $\begin{array}{c}2.06 \\
(1.60)\end{array}$ & $\begin{array}{c}2.13 \\
(1.62)\end{array}$ \\
\hline $\mathbf{N}_{1} \mathbf{K}_{3}$ & $\begin{array}{c}2.86 \\
(1.83)\end{array}$ & $\begin{array}{c}3.35 \\
(1.96)\end{array}$ & $\begin{array}{c}3.10 \\
(1.90)\end{array}$ & $\begin{array}{c}2.01 \\
(1.58)\end{array}$ & $\begin{array}{c}1.83 \\
(1.53)\end{array}$ & $\begin{array}{c}1.92 \\
(1.55)\end{array}$ \\
\hline $\mathbf{N}_{2} \mathbf{K}_{1}$ & $\begin{array}{c}3.64 \\
(2.04)\end{array}$ & $\begin{array}{c}3.91 \\
(2.10)\end{array}$ & $\begin{array}{c}3.77 \\
(2.07)\end{array}$ & $\begin{array}{c}2.51 \\
(1.73)\end{array}$ & $\begin{array}{c}2.38 \\
(1.70)\end{array}$ & $\begin{array}{c}2.44 \\
(1.72)\end{array}$ \\
\hline $\mathbf{N}_{2} \mathbf{K}_{2}$ & $\begin{array}{c}2.98 \\
(1.86)\end{array}$ & $\begin{array}{c}3.62 \\
(2.03)\end{array}$ & $\begin{array}{c}3.30 \\
(1.95)\end{array}$ & $\begin{array}{c}2.31 \\
(1.68)\end{array}$ & $\begin{array}{c}2.26 \\
(1.66)\end{array}$ & $\begin{array}{c}2.29 \\
(1.67)\end{array}$ \\
\hline $\mathbf{N}_{2} \mathbf{K}_{3}$ & $\begin{array}{c}2.49 \\
(1.73)\end{array}$ & $\begin{array}{c}3.49 \\
(2.00)\end{array}$ & $\begin{array}{c}2.99 \\
(1.86)\end{array}$ & $\begin{array}{c}1.99 \\
(1.58)\end{array}$ & $\begin{array}{c}2.12 \\
(1.62)\end{array}$ & $\begin{array}{c}2.05 \\
(1.60)\end{array}$ \\
\hline $\mathbf{N}_{3} \mathbf{K}_{1}$ & $\begin{array}{c}3.92 \\
(2.10)\end{array}$ & $\begin{array}{c}4.06 \\
(2.14)\end{array}$ & $\begin{array}{c}3.99 \\
(2.12)\end{array}$ & $\begin{array}{c}2.63 \\
(1.77)\end{array}$ & $\begin{array}{c}2.53 \\
(1.74)\end{array}$ & $\begin{array}{c}2.58 \\
(1.75)\end{array}$ \\
\hline $\mathbf{N}_{3} \mathbf{K}_{2}$ & $\begin{array}{c}3.20 \\
(1.92)\end{array}$ & $\begin{array}{c}3.74 \\
(2.06)\end{array}$ & $\begin{array}{c}3.47 \\
(1.99)\end{array}$ & $\begin{array}{c}2.35 \\
(1.69)\end{array}$ & $\begin{array}{c}2.39 \\
(1.70)\end{array}$ & $\begin{array}{c}2.37 \\
(1.69)\end{array}$ \\
\hline $\mathbf{N}_{3} K_{3}$ & $\begin{array}{c}2.48 \\
(1.72)\end{array}$ & $\begin{array}{c}3.51 \\
(2.00)\end{array}$ & $\begin{array}{c}2.99 \\
(1.86)\end{array}$ & $\begin{array}{c}2.22 \\
(1.65)\end{array}$ & $\begin{array}{c}2.13 \\
(1.62)\end{array}$ & $\begin{array}{c}2.17 \\
(1.63)\end{array}$ \\
\hline S.Em. \pm & 0.023 & 0.010 & 0.014 & 0.008 & 0.007 & 0.005 \\
\hline C.D. at 5\% & 0.066 & 0.028 & 0.039 & 0.018 & 0.020 & NS \\
\hline
\end{tabular}


Contd.....

\begin{tabular}{|c|c|c|c|c|c|c|}
\hline \multirow[t]{3}{*}{ Treatments } & \multicolumn{6}{|c|}{ Aphids (per 3 leaves) } \\
\hline & \multicolumn{3}{|c|}{70 DAS } & \multicolumn{3}{|c|}{90 DAS } \\
\hline & 2012-13 & 2013-14 & Pooled & 2012-13 & 2013-14 & Pooled \\
\hline $\mathbf{P}_{1} \mathbf{K}_{1}$ & $\begin{array}{c}3.60 \\
(2.02)\end{array}$ & $\begin{array}{c}3.82 \\
(2.08)\end{array}$ & $\begin{array}{c}3.71 \\
(2.05)\end{array}$ & $\begin{array}{c}2.44 \\
(1.72)\end{array}$ & $\begin{array}{c}2.34 \\
(1.69)\end{array}$ & $\begin{array}{c}2.39 \\
(1.70)\end{array}$ \\
\hline $\mathbf{P}_{1} \mathbf{K}_{2}$ & $\begin{array}{c}3.07 \\
(1.89)\end{array}$ & $\begin{array}{c}3.59 \\
(2.02)\end{array}$ & $\begin{array}{c}3.33 \\
(1.95)\end{array}$ & $\begin{array}{c}2.27 \\
(1.66)\end{array}$ & $\begin{array}{c}2.20 \\
(1.64)\end{array}$ & $\begin{array}{c}2.23 \\
(1.65)\end{array}$ \\
\hline $\mathbf{P}_{1} \mathbf{K}_{3}$ & $\begin{array}{c}2.69 \\
(1.79)\end{array}$ & $\begin{array}{c}3.39 \\
(1.97)\end{array}$ & $\begin{array}{c}3.04 \\
(1.88)\end{array}$ & $\begin{array}{c}2.03 \\
(1.59)\end{array}$ & $\begin{array}{c}2.02 \\
(1.59)\end{array}$ & $\begin{array}{c}2.02 \\
(1.59)\end{array}$ \\
\hline $\mathbf{P}_{2} \mathbf{K}_{1}$ & $\begin{array}{c}3.69 \\
(2.05)\end{array}$ & $\begin{array}{c}3.88 \\
(2.09)\end{array}$ & $\begin{array}{c}3.79 \\
(2.07)\end{array}$ & $\begin{array}{c}2.51 \\
(1.73)\end{array}$ & $\begin{array}{c}2.38 \\
(1.70)\end{array}$ & $\begin{array}{c}2.45 \\
(1.72)\end{array}$ \\
\hline $\mathbf{P}_{2} \mathbf{K}_{2}$ & $\begin{array}{c}3.13 \\
(1.90)\end{array}$ & $\begin{array}{c}3.65 \\
(2.04)\end{array}$ & $\begin{array}{c}3.39 \\
(1.97)\end{array}$ & $\begin{array}{c}2.30 \\
(1.67)\end{array}$ & $\begin{array}{c}2.28 \\
(1.67)\end{array}$ & $\begin{array}{c}2.29 \\
(1.67)\end{array}$ \\
\hline $\mathbf{P}_{2} \mathbf{K}_{3}$ & $\begin{array}{c}2.52 \\
(1.74)\end{array}$ & $\begin{array}{c}3.50 \\
(2.00) \\
\end{array}$ & $\begin{array}{c}3.01 \\
(1.87)\end{array}$ & $\begin{array}{c}2.11 \\
(1.61) \\
\end{array}$ & $\begin{array}{c}2.04 \\
(1.59)\end{array}$ & $\begin{array}{c}2.07 \\
(1.60) \\
\end{array}$ \\
\hline S.Em. \pm & 0.019 & 0.008 & 0.011 & 0.006 & 0.006 & 0.004 \\
\hline C.D. at 5\% & NS & NS & NS & NS & NS & NS \\
\hline $\mathbf{N}_{1} \mathbf{P}_{1} \mathbf{K}_{1}$ & $\begin{array}{c}3.33 \\
(1.96)\end{array}$ & $\begin{array}{c}3.58 \\
(2.02)\end{array}$ & $\begin{array}{c}3.45 \\
(1.99)\end{array}$ & $\begin{array}{c}2.27 \\
(1.67)\end{array}$ & $\begin{array}{c}2.15 \\
(1.63)\end{array}$ & $\begin{array}{c}2.21 \\
(1.65)\end{array}$ \\
\hline $\mathbf{N}_{1} \mathbf{P}_{1} \mathbf{K}_{2}$ & $\begin{array}{c}3.05 \\
(1.88)\end{array}$ & $\begin{array}{c}3.40 \\
(1.98)\end{array}$ & $\begin{array}{c}3.23 \\
(1.93)\end{array}$ & $\begin{array}{c}2.22 \\
(1.65)\end{array}$ & $\begin{array}{c}2.05 \\
(1.60)\end{array}$ & $\begin{array}{c}2.14 \\
(1.62)\end{array}$ \\
\hline $\mathbf{N}_{1} \mathbf{P}_{1} \mathbf{K}_{3}$ & $\begin{array}{c}2.89 \\
(1.84)\end{array}$ & $\begin{array}{c}3.25 \\
(1.94)\end{array}$ & $\begin{array}{c}3.07 \\
(1.89)\end{array}$ & $\begin{array}{c}2.06 \\
(1.60)\end{array}$ & $\begin{array}{c}1.89 \\
(1.54)\end{array}$ & $\begin{array}{c}1.97 \\
(1.57)\end{array}$ \\
\hline $\mathbf{N}_{1} \mathbf{P}_{2} \mathbf{K}_{1}$ & $\begin{array}{c}3.41 \\
(1.98)\end{array}$ & $\begin{array}{c}3.61 \\
(2.03)\end{array}$ & $\begin{array}{c}3.51 \\
(2.00)\end{array}$ & $\begin{array}{c}2.33 \\
(1.68)\end{array}$ & $\begin{array}{c}2.19 \\
(1.64)\end{array}$ & $\begin{array}{c}2.26 \\
(1.66)\end{array}$ \\
\hline $\mathbf{N}_{1} \mathbf{P}_{2} \mathbf{K}_{2}$ & $\begin{array}{c}3.20 \\
(1.92)\end{array}$ & $\begin{array}{c}3.59 \\
(2.02)\end{array}$ & $\begin{array}{c}3.40 \\
(1.97)\end{array}$ & $\begin{array}{c}2.16 \\
(1.63)\end{array}$ & $\begin{array}{c}2.07 \\
(1.60)\end{array}$ & $\begin{array}{c}2.12 \\
(1.62)\end{array}$ \\
\hline $\mathbf{N}_{1} \mathbf{P}_{2} \mathbf{K}_{3}$ & $\begin{array}{c}2.84 \\
(1.83)\end{array}$ & $\begin{array}{c}3.45 \\
(1.99)\end{array}$ & $\begin{array}{c}3.14 \\
(1.91)\end{array}$ & $\begin{array}{c}1.96 \\
(1.57)\end{array}$ & $\begin{array}{c}1.77 \\
(1.51)\end{array}$ & $\begin{array}{c}1.87 \\
(1.54)\end{array}$ \\
\hline $\mathbf{N}_{2} \mathbf{P}_{1} \mathbf{K}_{1}$ & $\begin{array}{c}3.58 \\
(2.02)\end{array}$ & $\begin{array}{c}3.84 \\
(2.08)\end{array}$ & $\begin{array}{c}3.71 \\
(2.05)\end{array}$ & $\begin{array}{c}2.45 \\
(1.72)\end{array}$ & $\begin{array}{c}2.36 \\
(1.69)\end{array}$ & $\begin{array}{c}2.41 \\
(1.71)\end{array}$ \\
\hline $\mathbf{N}_{2} \mathbf{P}_{1} \mathbf{K}_{2}$ & $\begin{array}{c}3.30 \\
(1.95)\end{array}$ & $\begin{array}{c}3.57 \\
(2.02)\end{array}$ & $\begin{array}{c}3.44 \\
(1.98)\end{array}$ & $\begin{array}{c}2.28 \\
(1.67)\end{array}$ & $\begin{array}{c}2.23 \\
(1.65)\end{array}$ & $\begin{array}{c}2.26 \\
(1.66)\end{array}$ \\
\hline $\mathbf{N}_{2} \mathbf{P}_{1} \mathbf{K}_{3}$ & $\begin{array}{c}2.76 \\
(1.81)\end{array}$ & $\begin{array}{c}3.47 \\
(1.99)\end{array}$ & $\begin{array}{c}3.12 \\
(1.90)\end{array}$ & $\begin{array}{c}1.88 \\
(1.54)\end{array}$ & $\begin{array}{c}2.15 \\
(1.63)\end{array}$ & $\begin{array}{c}2.02 \\
(1.59)\end{array}$ \\
\hline $\mathbf{N}_{2} \mathbf{P}_{2} \mathbf{K}_{1}$ & $\begin{array}{c}3.70 \\
(2.05)\end{array}$ & $\begin{array}{c}3.97 \\
(2.11)\end{array}$ & $\begin{array}{c}3.84 \\
(2.08)\end{array}$ & $\begin{array}{c}2.56 \\
(1.75)\end{array}$ & $\begin{array}{c}2.40 \\
(1.70)\end{array}$ & $\begin{array}{c}2.48 \\
(1.73)\end{array}$ \\
\hline $\mathbf{N}_{2} \mathbf{P}_{2} \mathbf{K}_{2}$ & $\begin{array}{c}2.66 \\
(1.78)\end{array}$ & $\begin{array}{c}3.67 \\
(2.04)\end{array}$ & $\begin{array}{c}3.17 \\
(1.91)\end{array}$ & $\begin{array}{c}2.34 \\
(1.68)\end{array}$ & $\begin{array}{c}2.29 \\
(1.67)\end{array}$ & $\begin{array}{c}2.31 \\
(1.68)\end{array}$ \\
\hline $\mathbf{N}_{2} \mathbf{P}_{2} \mathbf{K}_{3}$ & $\begin{array}{c}2.21 \\
(1.65)\end{array}$ & $\begin{array}{c}3.50 \\
(2.00)\end{array}$ & $\begin{array}{c}2.86 \\
(1.82)\end{array}$ & $\begin{array}{c}2.09 \\
(1.61)\end{array}$ & $\begin{array}{c}2.08 \\
(1.61)\end{array}$ & $\begin{array}{c}2.08 \\
(1.61)\end{array}$ \\
\hline $\mathbf{N}_{3} \mathbf{P}_{1} \mathbf{K}_{1}$ & $\begin{array}{c}3.89 \\
(2.10)\end{array}$ & $\begin{array}{c}4.05 \\
(2.13)\end{array}$ & $\begin{array}{c}3.97 \\
(2.11)\end{array}$ & $\begin{array}{c}2.60 \\
(1.76)\end{array}$ & $\begin{array}{c}2.51 \\
(1.74)\end{array}$ & $\begin{array}{c}2.56 \\
(1.75)\end{array}$ \\
\hline $\mathbf{N}_{3} \mathbf{P}_{1} \mathbf{K}_{2}$ & $\begin{array}{c}2.87 \\
(1.83)\end{array}$ & $\begin{array}{c}3.81 \\
(2.08)\end{array}$ & $\begin{array}{c}3.34 \\
(1.95)\end{array}$ & $\begin{array}{c}2.30 \\
(1.67)\end{array}$ & $\begin{array}{c}2.31 \\
(1.68)\end{array}$ & $\begin{array}{c}2.31 \\
(1.67)\end{array}$ \\
\hline $\mathbf{N}_{3} \mathbf{P}_{1} \mathbf{K}_{3}$ & $\begin{array}{c}2.43 \\
(1.71)\end{array}$ & $\begin{array}{c}3.45 \\
(1.99)\end{array}$ & $\begin{array}{c}2.94 \\
(1.85)\end{array}$ & $\begin{array}{c}2.14 \\
(1.63)\end{array}$ & $\begin{array}{c}2.01 \\
(1.58)\end{array}$ & $\begin{array}{c}2.08 \\
(1.61)\end{array}$ \\
\hline $\mathbf{N}_{3} \mathbf{P}_{2} \mathbf{K}_{1}$ & $\begin{array}{c}3.96 \\
(2.11)\end{array}$ & $\begin{array}{c}4.07 \\
(2.14)\end{array}$ & $\begin{array}{c}4.01 \\
(2.12)\end{array}$ & $\begin{array}{c}2.65 \\
(1.77)\end{array}$ & $\begin{array}{c}2.55 \\
(1.75)\end{array}$ & $\begin{array}{c}2.60 \\
(1.76)\end{array}$ \\
\hline $\mathbf{N}_{3} \mathbf{P}_{2} \mathbf{K}_{2}$ & $\begin{array}{c}3.54 \\
(2.01)\end{array}$ & $\begin{array}{c}3.68 \\
(2.04)\end{array}$ & $\begin{array}{c}3.61 \\
(2.03)\end{array}$ & $\begin{array}{c}2.41 \\
(1.70)\end{array}$ & $\begin{array}{c}2.47 \\
(1.72)\end{array}$ & $\begin{array}{c}2.44 \\
(1.71)\end{array}$ \\
\hline $\mathbf{N}_{3} \mathbf{P}_{2} \mathbf{K}_{3}$ & $\begin{array}{c}2.53 \\
(1.74)\end{array}$ & $\begin{array}{c}3.56 \\
(2.02)\end{array}$ & $\begin{array}{c}3.05 \\
(1.88)\end{array}$ & $\begin{array}{c}2.29 \\
(1.67)\end{array}$ & $\begin{array}{c}2.26 \\
(1.66)\end{array}$ & $\begin{array}{c}2.27 \\
(1.66)\end{array}$ \\
\hline S.Em. \pm & 0.032 & 0.014 & 0.019 & 0.011 & 0.010 & 0.007 \\
\hline C.D. at 5\% & NS & NS & NS & NS & NS & NS \\
\hline Control & $\begin{array}{c}4.25 \\
(2.18)\end{array}$ & $\begin{array}{c}4.96 \\
(2.34)\end{array}$ & $\begin{array}{c}4.61 \\
(2.26)\end{array}$ & $\begin{array}{c}2.63 \\
(1.77)\end{array}$ & $\begin{array}{c}2.71 \\
(1.79)\end{array}$ & $\begin{array}{c}2.67 \\
(1.78)\end{array}$ \\
\hline S.Em. \pm & 0.032 & 0.014 & 0.019 & 0.011 & 0.009 & 0.007 \\
\hline C.D. at $5 \%$ & 0.091 & 0.039 & 0.054 & 0.031 & 0.027 & 0.019 \\
\hline
\end{tabular}


Table.4 Shoot weevil population (per 10 plants) in Bt cotton as influenced by different levels of NPK in Alfisol

\begin{tabular}{|c|c|c|c|c|c|c|}
\hline \multirow[t]{3}{*}{ Treatments } & \multicolumn{6}{|c|}{ Shoot weevil (per 10 plants) } \\
\hline & \multicolumn{3}{|c|}{70 DAS } & \multicolumn{3}{|c|}{90 DAS } \\
\hline & 2012-13 & 2013-14 & Pooled & 2012-13 & 2013-14 & Pooled \\
\hline $\mathbf{N}_{1}$ & $\begin{array}{c}3.46 \\
(1.99)\end{array}$ & $\begin{array}{c}3.67 \\
(2.04)\end{array}$ & $\begin{array}{c}3.57 \\
(2.02)\end{array}$ & $\begin{array}{c}3.37 \\
(1.97)\end{array}$ & $\begin{array}{c}3.68 \\
(2.04)\end{array}$ & $\begin{array}{c}3.53 \\
(2.01)\end{array}$ \\
\hline $\mathbf{N}_{2}$ & $\begin{array}{c}3.94 \\
(2.11)\end{array}$ & $\begin{array}{c}3.75 \\
(2.06)\end{array}$ & $\begin{array}{c}3.84 \\
(2.08)\end{array}$ & $\begin{array}{c}3.41 \\
(1.98)\end{array}$ & $\begin{array}{l}3.70 \\
(2.05)\end{array}$ & $\begin{array}{c}3.55 \\
(2.01)\end{array}$ \\
\hline $\mathbf{N}_{3}$ & $\begin{array}{c}4.18 \\
(2.16)\end{array}$ & $\begin{array}{c}3.87 \\
(2.09)\end{array}$ & $\begin{array}{c}4.03 \\
(2.13)\end{array}$ & $\begin{array}{c}3.49 \\
(2.00)\end{array}$ & $\begin{array}{c}3.77 \\
(2.07)\end{array}$ & $\begin{array}{c}3.63 \\
(2.03)\end{array}$ \\
\hline S.Em. \pm & 0.004 & 0.004 & 0.003 & 0.006 & 0.004 & 0.003 \\
\hline C.D. at 5\% & 0.012 & 0.013 & 0.008 & 0.016 & 0.012 & 0.010 \\
\hline $\mathbf{P}_{1}$ & $\begin{array}{c}3.83 \\
(2.08)\end{array}$ & $\begin{array}{c}3.74 \\
(2.06)\end{array}$ & $\begin{array}{c}3.79 \\
(2.07)\end{array}$ & $\begin{array}{c}3.42 \\
(1.98)\end{array}$ & $\begin{array}{c}3.68 \\
(2.04)\end{array}$ & $\begin{array}{c}3.55 \\
(2.01) \\
\end{array}$ \\
\hline $\mathbf{P}_{2}$ & $\begin{array}{c}3.89 \\
(2.09)\end{array}$ & $\begin{array}{c}3.79 \\
(2.07)\end{array}$ & $\begin{array}{c}3.84 \\
(2.08)\end{array}$ & $\begin{array}{c}3.43 \\
(1.98)\end{array}$ & $\begin{array}{c}3.75 \\
(2.06)\end{array}$ & $\begin{array}{c}3.59 \\
(2.02)\end{array}$ \\
\hline S.Em. \pm & 0.003 & 0.004 & 0.002 & 0.005 & 0.003 & 0.003 \\
\hline C.D. at 5\% & 0.010 & 0.011 & 0.007 & NS & 0.010 & 0.008 \\
\hline $\mathbf{K}_{1}$ & $\begin{array}{c}3.93 \\
(2.10)\end{array}$ & $\begin{array}{c}3.99 \\
(2.12)\end{array}$ & $\begin{array}{c}3.96 \\
(2.11)\end{array}$ & $\begin{array}{c}3.66 \\
(2.04)\end{array}$ & $\begin{array}{c}3.96 \\
(2.11)\end{array}$ & $\begin{array}{c}3.81 \\
(2.08)\end{array}$ \\
\hline $\mathbf{K}_{2}$ & $\begin{array}{c}3.88 \\
(2.09)\end{array}$ & $\begin{array}{c}3.78 \\
(2.07)\end{array}$ & $\begin{array}{l}3.83 \\
(2.08)\end{array}$ & $\begin{array}{c}3.42 \\
(1.98)\end{array}$ & $\begin{array}{c}3.73 \\
(2.06)\end{array}$ & $\begin{array}{c}3.57 \\
(2.02)\end{array}$ \\
\hline $\mathbf{K}_{3}$ & $\begin{array}{c}3.77 \\
(2.07)\end{array}$ & $\begin{array}{c}3.52 \\
(2.01)\end{array}$ & $\begin{array}{c}3.65 \\
(2.04)\end{array}$ & $\begin{array}{c}3.19 \\
(1.92)\end{array}$ & $\begin{array}{c}3.45 \\
(1.99)\end{array}$ & $\begin{array}{c}3.32 \\
(1.95)\end{array}$ \\
\hline S.Em. \pm & 0.004 & 0.004 & 0.003 & 0.006 & 0.004 & 0.003 \\
\hline C.D. at 5\% & 0.012 & 0.013 & 0.008 & 0.016 & 0.012 & 0.010 \\
\hline $\mathbf{N}_{1} \mathbf{P}_{1}$ & $\begin{array}{c}3.41 \\
(1.98)\end{array}$ & $\begin{array}{c}3.66 \\
(2.04)\end{array}$ & $\begin{array}{c}3.53 \\
(2.01)\end{array}$ & $\begin{array}{c}3.36 \\
(1.96)\end{array}$ & $\begin{array}{c}3.67 \\
(2.04)\end{array}$ & $\begin{array}{c}3.52 \\
(2.00)\end{array}$ \\
\hline $\mathbf{N}_{1} \mathbf{P}_{2}$ & $\begin{array}{c}3.51 \\
(2.00)\end{array}$ & $\begin{array}{c}3.69 \\
(2.05)\end{array}$ & $\begin{array}{l}3.60 \\
(2.02)\end{array}$ & $\begin{array}{c}3.39 \\
(1.97)\end{array}$ & $\begin{array}{c}3.68 \\
(2.04)\end{array}$ & $\begin{array}{c}3.54 \\
(2.01)\end{array}$ \\
\hline $\mathbf{N}_{2} \mathbf{P}_{1}$ & $\begin{array}{c}3.91 \\
(2.10)\end{array}$ & $\begin{array}{c}3.72 \\
(2.05)\end{array}$ & $\begin{array}{c}3.82 \\
(2.08)\end{array}$ & $\begin{array}{c}3.41 \\
(1.98)\end{array}$ & $\begin{array}{c}3.65 \\
(2.04)\end{array}$ & $\begin{array}{c}3.53 \\
(2.01)\end{array}$ \\
\hline $\mathbf{N}_{2} \mathbf{P}_{2}$ & $\begin{array}{l}3.96 \\
(2.11)\end{array}$ & $\begin{array}{l}3.77 \\
(2.07)\end{array}$ & $\begin{array}{c}3.87 \\
(2.09)\end{array}$ & $\begin{array}{c}3.40 \\
(1.97)\end{array}$ & $\begin{array}{c}3.74 \\
(2.06)\end{array}$ & $\begin{array}{c}3.57 \\
(2.02)\end{array}$ \\
\hline $\mathbf{N}_{3} \mathbf{P}_{1}$ & $\begin{array}{l}4.18 \\
(2.16)\end{array}$ & $\begin{array}{c}3.84 \\
(2.08)\end{array}$ & $\begin{array}{l}4.01 \\
(2.12)\end{array}$ & $\begin{array}{c}3.48 \\
(1.99)\end{array}$ & $\begin{array}{c}3.72 \\
(2.05)\end{array}$ & $\begin{array}{l}3.60 \\
(2.02)\end{array}$ \\
\hline $\mathbf{N}_{3} \mathbf{P}_{2}$ & $\begin{array}{c}4.19 \\
(2.17)\end{array}$ & $\begin{array}{c}3.91 \\
(2.10)\end{array}$ & $\begin{array}{l}4.05 \\
(2.13)\end{array}$ & $\begin{array}{c}3.50 \\
(2.00)\end{array}$ & $\begin{array}{c}3.82 \\
(2.08)\end{array}$ & $\begin{array}{c}3.66 \\
(2.04)\end{array}$ \\
\hline S.Em. \pm & 0.006 & 0.006 & 0.004 & 0.008 & 0.006 & 0.005 \\
\hline C.D. at $5 \%$ & NS & NS & NS & NS & NS & NS \\
\hline $\mathbf{N}_{1} K_{1}$ & $\begin{array}{c}3.51 \\
(2.00)\end{array}$ & $\begin{array}{c}3.85 \\
(2.09)\end{array}$ & $\begin{array}{c}3.68 \\
(2.04)\end{array}$ & $\begin{array}{c}3.57 \\
(2.02)\end{array}$ & $\begin{array}{c}3.87 \\
(2.09)\end{array}$ & $\begin{array}{c}3.72 \\
(2.05)\end{array}$ \\
\hline $\mathbf{N}_{1} \mathbf{K}_{2}$ & $\begin{array}{c}3.46 \\
(1.99)\end{array}$ & $\begin{array}{c}3.67 \\
(2.04)\end{array}$ & $\begin{array}{c}3.57 \\
(2.02)\end{array}$ & $\begin{array}{c}3.37 \\
(1.97)\end{array}$ & $\begin{array}{c}3.70 \\
(2.05)\end{array}$ & $\begin{array}{c}3.53 \\
(2.01)\end{array}$ \\
\hline $\mathbf{N}_{1} \mathbf{K}_{3}$ & $\begin{array}{c}3.41 \\
(1.98)\end{array}$ & $\begin{array}{c}3.50 \\
(2.00)\end{array}$ & $\begin{array}{c}3.46 \\
(1.99)\end{array}$ & $\begin{array}{c}3.18 \\
(1.92)\end{array}$ & $\begin{array}{c}3.47 \\
(1.99)\end{array}$ & $\begin{array}{c}3.32 \\
(1.96)\end{array}$ \\
\hline $\mathbf{N}_{2} \mathbf{K}_{1}$ & $\begin{array}{c}4.04 \\
(2.13)\end{array}$ & $\begin{array}{c}3.99 \\
(2.12) \\
\end{array}$ & $\begin{array}{c}4.01 \\
(2.12)\end{array}$ & $\begin{array}{c}3.66 \\
(2.04) \\
\end{array}$ & $\begin{array}{c}3.95 \\
(2.11)\end{array}$ & $\begin{array}{c}3.80 \\
(2.07) \\
\end{array}$ \\
\hline $\mathbf{N}_{2} \mathbf{K}_{2}$ & $\begin{array}{c}3.96 \\
(2.11)\end{array}$ & $\begin{array}{c}3.78 \\
(2.07)\end{array}$ & $\begin{array}{c}3.87 \\
(2.09)\end{array}$ & $\begin{array}{c}3.41 \\
(1.98)\end{array}$ & $\begin{array}{c}3.72 \\
(2.05)\end{array}$ & $\begin{array}{c}3.57 \\
(2.02)\end{array}$ \\
\hline $\mathbf{N}_{2} \mathbf{K}_{3}$ & $\begin{array}{c}3.82 \\
(2.08)\end{array}$ & $\begin{array}{c}3.47 \\
(1.99)\end{array}$ & $\begin{array}{c}3.64 \\
(2.03)\end{array}$ & $\begin{array}{c}3.15 \\
(1.91)\end{array}$ & $\begin{array}{c}3.42 \\
(1.98)\end{array}$ & $\begin{array}{c}3.28 \\
(1.94)\end{array}$ \\
\hline $\mathbf{N}_{3} \mathbf{K}_{1}$ & $\begin{array}{c}4.24 \\
(2.18)\end{array}$ & $\begin{array}{c}4.13 \\
(2.15)\end{array}$ & $\begin{array}{c}4.19 \\
(2.16)\end{array}$ & $\begin{array}{c}3.75 \\
(2.06)\end{array}$ & $\begin{array}{c}4.08 \\
(2.14)\end{array}$ & $\begin{array}{c}3.91 \\
(2.10)\end{array}$ \\
\hline $\mathbf{N}_{3} \mathbf{K}_{2}$ & $\begin{array}{c}4.21 \\
(2.17)\end{array}$ & $\begin{array}{c}3.90 \\
(2.10)\end{array}$ & $\begin{array}{c}4.05 \\
(2.13)\end{array}$ & $\begin{array}{c}3.48 \\
(2.00)\end{array}$ & $\begin{array}{c}3.76 \\
(2.06)\end{array}$ & $\begin{array}{c}3.62 \\
(2.03)\end{array}$ \\
\hline $\mathbf{N}_{3} \mathbf{K}_{3}$ & $\begin{array}{c}4.10 \\
(2.14)\end{array}$ & $\begin{array}{c}3.60 \\
(2.02)\end{array}$ & $\begin{array}{c}3.85 \\
(2.08)\end{array}$ & $\begin{array}{c}3.24 \\
(1.93)\end{array}$ & $\begin{array}{c}3.47 \\
(1.99)\end{array}$ & $\begin{array}{c}3.36 \\
(1.96)\end{array}$ \\
\hline S.Em. \pm & 0.007 & 0.008 & 0.005 & 0.010 & 0.007 & 0.006 \\
\hline C.D. at $5 \%$ & NS & 0.022 & 0.014 & NS & 0.021 & 0.017 \\
\hline
\end{tabular}


Contd.

\begin{tabular}{|c|c|c|c|c|c|c|}
\hline \multirow[t]{3}{*}{ Treatments } & \multicolumn{6}{|c|}{ Shoot weevil (per 10 plants) } \\
\hline & \multicolumn{3}{|c|}{70 DAS } & \multicolumn{3}{|c|}{90 DAS } \\
\hline & 2012-13 & 2013-14 & Pooled & 2012-13 & 2013-14 & Pooled \\
\hline $\mathbf{P}_{1} \mathbf{K}_{1}$ & $\begin{array}{c}3.89 \\
(2.09)\end{array}$ & $\begin{array}{c}3.96 \\
(2.11)\end{array}$ & $\begin{array}{c}3.92 \\
(2.10)\end{array}$ & $\begin{array}{c}3.64 \\
(2.03)\end{array}$ & $\begin{array}{c}3.95 \\
(2.11)\end{array}$ & $\begin{array}{c}3.80 \\
(2.07)\end{array}$ \\
\hline $\mathbf{P}_{1} \mathbf{K}_{2}$ & $\begin{array}{c}3.84 \\
(2.08)\end{array}$ & $\begin{array}{c}3.75 \\
(2.06)\end{array}$ & $\begin{array}{c}3.79 \\
(2.07) \\
\end{array}$ & $\begin{array}{c}3.43 \\
(1.98)\end{array}$ & $\begin{array}{c}3.70 \\
(2.05)\end{array}$ & $\begin{array}{c}3.57 \\
(2.02)\end{array}$ \\
\hline $\mathbf{P}_{1} \mathbf{K}_{3}$ & $\begin{array}{c}3.77 \\
(2.07) \\
\end{array}$ & $\begin{array}{c}3.51 \\
(2.00)\end{array}$ & $\begin{array}{c}3.64 \\
(2.03)\end{array}$ & $\begin{array}{c}3.18 \\
(1.92)\end{array}$ & $\begin{array}{c}3.39 \\
(1.97)\end{array}$ & $\begin{array}{c}3.28 \\
(1.94)\end{array}$ \\
\hline $\mathbf{P}_{2} \mathbf{K}_{1}$ & $\begin{array}{c}3.98 \\
(2.11)\end{array}$ & $\begin{array}{c}4.02 \\
(2.13)\end{array}$ & $\begin{array}{c}4.00 \\
(2.12)\end{array}$ & $\begin{array}{c}3.68 \\
(2.04)\end{array}$ & $\begin{array}{c}3.97 \\
(2.11)\end{array}$ & $\begin{array}{c}3.83 \\
(2.08)\end{array}$ \\
\hline $\mathbf{P}_{2} \mathbf{K}_{2}$ & $\begin{array}{c}3.91 \\
(2.10)\end{array}$ & $\begin{array}{c}3.81 \\
(2.08)\end{array}$ & $\begin{array}{c}3.86 \\
(2.09)\end{array}$ & $\begin{array}{c}3.41 \\
(1.98)\end{array}$ & $\begin{array}{c}3.75 \\
(2.06)\end{array}$ & $\begin{array}{c}3.58 \\
(2.02)\end{array}$ \\
\hline $\mathbf{P}_{2} \mathbf{K}_{3}$ & $\begin{array}{c}3.78 \\
(2.07) \\
\end{array}$ & $\begin{array}{c}3.54 \\
(2.01) \\
\end{array}$ & $\begin{array}{c}3.66 \\
(2.04) \\
\end{array}$ & $\begin{array}{c}3.20 \\
(1.92) \\
\end{array}$ & $\begin{array}{c}3.52 \\
(2.00) \\
\end{array}$ & $\begin{array}{c}3.36 \\
(1.96) \\
\end{array}$ \\
\hline S.Em. \pm & 0.006 & 0.006 & 0.004 & 0.008 & 0.006 & 0.005 \\
\hline C.D. at $5 \%$ & NS & NS & NS & NS & NS & NS \\
\hline $\mathbf{N}_{1} \mathbf{P}_{1} \mathbf{K}_{1}$ & $\begin{array}{c}3.44 \\
(1.98)\end{array}$ & $\begin{array}{c}3.80 \\
(2.07)\end{array}$ & $\begin{array}{c}3.62 \\
(2.03)\end{array}$ & $\begin{array}{c}3.53 \\
(2.01)\end{array}$ & $\begin{array}{c}3.86 \\
(2.09)\end{array}$ & $\begin{array}{c}3.70 \\
(2.05)\end{array}$ \\
\hline $\mathbf{N}_{1} \mathbf{P}_{1} \mathbf{K}_{2}$ & $\begin{array}{c}3.42 \\
(1.98)\end{array}$ & $\begin{array}{c}3.66 \\
(2.04)\end{array}$ & $\begin{array}{c}3.54 \\
(2.01)\end{array}$ & $\begin{array}{c}3.39 \\
(1.97)\end{array}$ & $\begin{array}{c}3.71 \\
(2.05)\end{array}$ & $\begin{array}{c}3.55 \\
(2.01)\end{array}$ \\
\hline $\mathbf{N}_{1} \mathbf{P}_{1} \mathbf{K}_{3}$ & $\begin{array}{c}3.37 \\
(1.97)\end{array}$ & $\begin{array}{c}3.52 \\
(2.00)\end{array}$ & $\begin{array}{c}3.44 \\
(1.99)\end{array}$ & $\begin{array}{c}3.14 \\
(1.91)\end{array}$ & $\begin{array}{c}3.45 \\
(1.99)\end{array}$ & $\begin{array}{c}3.30 \\
(1.95)\end{array}$ \\
\hline $\mathbf{N}_{1} \mathbf{P}_{2} \mathbf{K}_{1}$ & $\begin{array}{c}3.58 \\
(2.02) \\
\end{array}$ & $\begin{array}{c}3.90 \\
(2.10)\end{array}$ & $\begin{array}{c}3.74 \\
(2.06) \\
\end{array}$ & $\begin{array}{c}3.61 \\
(2.03)\end{array}$ & $\begin{array}{c}3.88 \\
(2.09) \\
\end{array}$ & $\begin{array}{c}3.74 \\
(2.06)\end{array}$ \\
\hline $\mathbf{N}_{1} \mathbf{P}_{2} \mathbf{K}_{2}$ & $\begin{array}{c}3.50 \\
(2.00)\end{array}$ & $\begin{array}{c}3.68 \\
(2.04)\end{array}$ & $\begin{array}{c}3.59 \\
(2.02)\end{array}$ & $\begin{array}{c}3.34 \\
(1.96)\end{array}$ & $\begin{array}{c}3.69 \\
(2.05)\end{array}$ & $\begin{array}{c}3.52 \\
(2.00)\end{array}$ \\
\hline $\mathbf{N}_{1} \mathbf{P}_{2} \mathbf{K}_{3}$ & $\begin{array}{c}3.46 \\
(1.99)\end{array}$ & $\begin{array}{c}3.49 \\
(2.00)\end{array}$ & $\begin{array}{c}3.47 \\
(1.99)\end{array}$ & $\begin{array}{c}3.22 \\
(1.93)\end{array}$ & $\begin{array}{c}3.48 \\
(1.99)\end{array}$ & $\begin{array}{c}3.35 \\
(1.96)\end{array}$ \\
\hline $\mathbf{N}_{2} \mathbf{P}_{1} \mathbf{K}_{1}$ & $\begin{array}{c}4.00 \\
(2.12)\end{array}$ & $\begin{array}{c}3.96 \\
(2.11)\end{array}$ & $\begin{array}{c}3.98 \\
(2.12)\end{array}$ & $\begin{array}{c}3.64 \\
(2.04)\end{array}$ & $\begin{array}{c}3.93 \\
(2.10)\end{array}$ & $\begin{array}{c}3.79 \\
(2.07)\end{array}$ \\
\hline $\mathbf{N}_{2} \mathbf{P}_{1} \mathbf{K}_{2}$ & $\begin{array}{c}3.90 \\
(2.10)\end{array}$ & $\begin{array}{c}3.76 \\
(2.06)\end{array}$ & $\begin{array}{c}3.83 \\
(2.08)\end{array}$ & $\begin{array}{c}3.39 \\
(1.97)\end{array}$ & $\begin{array}{c}3.68 \\
(2.04)\end{array}$ & $\begin{array}{c}3.53 \\
(2.01)\end{array}$ \\
\hline $\mathbf{N}_{2} \mathbf{P}_{1} \mathbf{K}_{3}$ & $\begin{array}{c}3.84 \\
(2.08) \\
\end{array}$ & $\begin{array}{c}3.44 \\
(1.98)\end{array}$ & $\begin{array}{c}3.64 \\
(2.03) \\
\end{array}$ & $\begin{array}{c}3.19 \\
(1.92)\end{array}$ & $\begin{array}{c}3.35 \\
(1.96) \\
\end{array}$ & $\begin{array}{c}3.27 \\
(1.94)\end{array}$ \\
\hline $\mathbf{N}_{2} \mathbf{P}_{2} \mathbf{K}_{1}$ & $\begin{array}{c}4.08 \\
(2.14)\end{array}$ & $\begin{array}{c}4.01 \\
(2.12)\end{array}$ & $\begin{array}{c}4.05 \\
(2.13)\end{array}$ & $\begin{array}{c}3.67 \\
(2.04)\end{array}$ & $\begin{array}{c}3.97 \\
(2.11)\end{array}$ & $\begin{array}{c}3.82 \\
(2.08)\end{array}$ \\
\hline $\mathbf{N}_{2} \mathbf{P}_{2} \mathbf{K}_{2}$ & $\begin{array}{c}4.01 \\
(2.12)\end{array}$ & $\begin{array}{c}3.80 \\
(2.07)\end{array}$ & $\begin{array}{c}3.91 \\
(2.10)\end{array}$ & $\begin{array}{c}3.43 \\
(1.98)\end{array}$ & $\begin{array}{c}3.76 \\
(2.06)\end{array}$ & $\begin{array}{c}3.60 \\
(2.02)\end{array}$ \\
\hline $\mathbf{N}_{2} \mathbf{P}_{2} \mathbf{K}_{3}$ & $\begin{array}{c}3.80 \\
(2.07)\end{array}$ & $\begin{array}{c}3.50 \\
(2.00)\end{array}$ & $\begin{array}{c}3.65 \\
(2.04)\end{array}$ & $\begin{array}{c}3.11 \\
(1.90)\end{array}$ & $\begin{array}{c}3.49 \\
(2.00)\end{array}$ & $\begin{array}{c}3.30 \\
(1.95)\end{array}$ \\
\hline $\mathbf{N}_{3} \mathbf{P}_{1} \mathbf{K}_{1}$ & $\begin{array}{c}4.23 \\
(2.17)\end{array}$ & $\begin{array}{c}4.11 \\
(2.15)\end{array}$ & $\begin{array}{c}4.17 \\
(2.16)\end{array}$ & $\begin{array}{c}3.73 \\
(2.06)\end{array}$ & $\begin{array}{c}4.08 \\
(2.14)\end{array}$ & $\begin{array}{c}3.91 \\
(2.10)\end{array}$ \\
\hline $\mathbf{N}_{\mathbf{3}} \mathbf{P}_{1} \mathbf{K}_{2}$ & $\begin{array}{c}4.19 \\
(2.17) \\
\end{array}$ & $\begin{array}{c}3.83 \\
(2.08)\end{array}$ & $\begin{array}{c}4.01 \\
(2.12)\end{array}$ & $\begin{array}{c}3.51 \\
(2.00)\end{array}$ & $\begin{array}{c}3.71 \\
(2.05)\end{array}$ & $\begin{array}{c}3.61 \\
(2.03)\end{array}$ \\
\hline $\mathbf{N}_{3} \mathbf{P}_{1} \mathbf{K}_{3}$ & $\begin{array}{c}4.11 \\
(2.15)\end{array}$ & $\begin{array}{c}3.57 \\
(2.02)\end{array}$ & $\begin{array}{c}3.84 \\
(2.08)\end{array}$ & $\begin{array}{c}3.21 \\
(1.93)\end{array}$ & $\begin{array}{c}3.36 \\
(1.96)\end{array}$ & $\begin{array}{c}3.28 \\
(1.94)\end{array}$ \\
\hline $\mathbf{N}_{3} \mathbf{P}_{2} \mathbf{K}_{1}$ & $\begin{array}{c}4.26 \\
(2.18)\end{array}$ & $\begin{array}{c}4.15 \\
(2.16)\end{array}$ & $\begin{array}{c}4.21 \\
(2.17)\end{array}$ & $\begin{array}{c}3.76 \\
(2.06)\end{array}$ & $\begin{array}{c}4.08 \\
(2.14)\end{array}$ & $\begin{array}{c}3.92 \\
(2.10)\end{array}$ \\
\hline $\mathbf{N}_{3} \mathbf{P}_{2} \mathbf{K}_{2}$ & $\begin{array}{c}4.23 \\
(2.17)\end{array}$ & $\begin{array}{c}3.96 \\
(2.11)\end{array}$ & $\begin{array}{c}4.10 \\
(2.14)\end{array}$ & $\begin{array}{c}3.46 \\
(1.99)\end{array}$ & $\begin{array}{c}3.80 \\
(2.07)\end{array}$ & $\begin{array}{c}3.63 \\
(2.03)\end{array}$ \\
\hline $\mathbf{N}_{\mathbf{3}} \mathbf{P}_{2} \mathbf{K}_{3}$ & $\begin{array}{c}4.08 \\
(2.14)\end{array}$ & $\begin{array}{c}3.62 \\
(2.03)\end{array}$ & $\begin{array}{c}3.85 \\
(2.08)\end{array}$ & $\begin{array}{c}3.27 \\
(1.94)\end{array}$ & $\begin{array}{c}3.58 \\
(2.02)\end{array}$ & $\begin{array}{c}3.43 \\
(1.98)\end{array}$ \\
\hline S.Em. \pm & 0.010 & 0.011 & 0.007 & 0.014 & 0.010 & 0.008 \\
\hline C.D. at $5 \%$ & NS & NS & NS & NS & NS & NS \\
\hline Control & $\begin{array}{c}5.11 \\
(2.37)\end{array}$ & $\begin{array}{c}4.97 \\
(2.34)\end{array}$ & $\begin{array}{c}5.04 \\
(2.35)\end{array}$ & $\begin{array}{c}4.88 \\
(2.32)\end{array}$ & $\begin{array}{c}4.50 \\
(2.23)\end{array}$ & $\begin{array}{c}4.69 \\
(2.28)\end{array}$ \\
\hline S.Em. \pm & 0.010 & 0.011 & 0.007 & 0.013 & 0.014 & 0.010 \\
\hline C.D. at $5 \%$ & 0.028 & 0.031 & 0.020 & 0.038 & 0.040 & 0.027 \\
\hline
\end{tabular}


Table.5 Mirid bug and midge population (per 10 squares) in Bt cotton as influenced by different levels of NPK in Alfisol

\begin{tabular}{|c|c|c|c|}
\hline \multirow[t]{3}{*}{ Treatments } & \multicolumn{2}{|c|}{ Mirid bug (per 10 squares) } & \multirow[t]{2}{*}{ Midge (per 10 squares) } \\
\hline & \multicolumn{2}{|c|}{ 2013-14 } & \\
\hline & 90 DAS & 110 DAS & 110 DAS \\
\hline $\mathbf{N}_{1}$ & $\begin{array}{c}2.34 \\
(1.69)\end{array}$ & $\begin{array}{c}3.70 \\
(2.05)\end{array}$ & $\begin{array}{c}1.85 \\
(1.53)\end{array}$ \\
\hline $\mathbf{N}_{2}$ & $\begin{array}{c}2.36 \\
(1.69)\end{array}$ & $\begin{array}{c}3.67 \\
(2.04)\end{array}$ & $\begin{array}{c}1.86 \\
(1.53)\end{array}$ \\
\hline $\mathbf{N}_{3}$ & $\begin{array}{c}2.40 \\
(1.70)\end{array}$ & $\begin{array}{c}3.74 \\
(2.06)\end{array}$ & $\begin{array}{c}1.85 \\
(1.53)\end{array}$ \\
\hline S.Em. \pm & 0.004 & 0.005 & 0.003 \\
\hline C.D. at $5 \%$ & 0.010 & NS & NS \\
\hline $\mathbf{P}_{1}$ & $\begin{array}{c}2.38 \\
(1.70)\end{array}$ & $\begin{array}{c}3.70 \\
(2.05)\end{array}$ & $\begin{array}{c}1.8 \\
(1.53)\end{array}$ \\
\hline $\mathbf{P}_{2}$ & $\begin{array}{c}2.36 \\
(1.69)\end{array}$ & $\begin{array}{c}3.71 \\
(2.05)\end{array}$ & $\begin{array}{c}1.85 \\
(1.53)\end{array}$ \\
\hline S.Em. \pm & 0.003 & 0.004 & 0.002 \\
\hline C.D. at $5 \%$ & NS & NS & NS \\
\hline $\mathbf{K}_{1}$ & $\begin{array}{c}2.40 \\
(1.70)\end{array}$ & $\begin{array}{c}3.76 \\
(2.06)\end{array}$ & $\begin{array}{c}1.86 \\
(1.54)\end{array}$ \\
\hline $\mathbf{K}_{2}$ & $\begin{array}{c}2.36 \\
(1.69)\end{array}$ & $\begin{array}{c}(2.00) \\
(2.09)\end{array}$ & $\begin{array}{c}1.85 \\
(1.53)\end{array}$ \\
\hline $\mathbf{K}_{\mathbf{3}}$ & $\begin{array}{c}2.34 \\
(1.68)\end{array}$ & $\begin{array}{c}3.67 \\
(2.04)\end{array}$ & $\begin{array}{c}1.85 \\
(1.53)\end{array}$ \\
\hline S.Em. \pm & 0.004 & 0.005 & 0.003 \\
\hline C.D. at $5 \%$ & 0.010 & 0.014 & NS \\
\hline $\mathbf{N}_{1} \mathbf{P}_{1}$ & $\begin{array}{c}2.35 \\
(1.69)\end{array}$ & $\begin{array}{c}3.67 \\
(2.04)\end{array}$ & $\begin{array}{c}1.83 \\
(1.53)\end{array}$ \\
\hline $\mathbf{N}_{1} \mathbf{P}_{2}$ & $\begin{array}{c}2.33 \\
(1.68)\end{array}$ & $\begin{array}{c}3.73 \\
(2.06)\end{array}$ & $\begin{array}{c}1.86 \\
(1.54)\end{array}$ \\
\hline $\mathbf{N}_{2} \mathbf{P}_{1}$ & $\begin{array}{c}2.38 \\
(1.70)\end{array}$ & $\begin{array}{c}3.68 \\
(2.05)\end{array}$ & $\begin{array}{c}1.86 \\
(1.54)\end{array}$ \\
\hline $\mathbf{N}_{2} \mathbf{P}_{2}$ & $\begin{array}{c}2.34 \\
(1.69)\end{array}$ & $\begin{array}{c}3.66 \\
(2.04)\end{array}$ & $\begin{array}{c}1.85 \\
(1.53)\end{array}$ \\
\hline $\mathbf{N}_{3} \mathbf{P}_{1}$ & $\begin{array}{c}2.40 \\
(1.70)\end{array}$ & $\begin{array}{c}3.75 \\
(2.06)\end{array}$ & $\begin{array}{c}1.86 \\
(1.54)\end{array}$ \\
\hline $\mathbf{N}_{3} \mathbf{P}_{2}$ & $\begin{array}{c}2.40 \\
(1.70)\end{array}$ & $\begin{array}{c}3.73 \\
(2.06)\end{array}$ & $\begin{array}{c}1.84 \\
(1.53)\end{array}$ \\
\hline S.Em. \pm & 0.005 & 0.007 & 0.004 \\
\hline C.D. at $5 \%$ & NS & NS & NS \\
\hline $\mathbf{N}_{1} \mathbf{K}_{1}$ & $\begin{array}{c}2.35 \\
(1.69)\end{array}$ & $\begin{array}{c}3.72 \\
(2.05)\end{array}$ & $\begin{array}{c}1.84 \\
(1.53)\end{array}$ \\
\hline $\mathbf{N}_{1} \mathbf{K}_{2}$ & $\begin{array}{c}2.34 \\
(1.68)\end{array}$ & $\begin{array}{c}3.68 \\
(2.05)\end{array}$ & $\begin{array}{c}1.85 \\
(1.53)\end{array}$ \\
\hline $\mathbf{N}_{1} \mathbf{K}_{3}$ & $\begin{array}{c}2.34 \\
(1.68)\end{array}$ & $\begin{array}{c}3.70 \\
(2.05)\end{array}$ & $\begin{array}{c}1.85 \\
(1.53)\end{array}$ \\
\hline $\mathbf{N}_{2} \mathbf{K}_{1}$ & $\begin{array}{c}2.41 \\
(1.70)\end{array}$ & $\begin{array}{c}3.77 \\
(2.07)\end{array}$ & $\begin{array}{c}1.87 \\
(1.54)\end{array}$ \\
\hline $\mathbf{N}_{2} \mathbf{K}_{2}$ & $\begin{array}{c}2.36 \\
(1.69)\end{array}$ & $\begin{array}{c}3.64 \\
(2.04)\end{array}$ & $\begin{array}{c}1.86 \\
(1.54)\end{array}$ \\
\hline $\mathbf{N}_{2} \mathbf{K}_{3}$ & $\begin{array}{c}2.32 \\
(1.68)\end{array}$ & $\begin{array}{c}3.61 \\
(2.03)\end{array}$ & $\begin{array}{c}1.84 \\
(1.53)\end{array}$ \\
\hline $\mathbf{N}_{3} \mathbf{K}_{1}$ & $\begin{array}{c}2.44 \\
(1.72)\end{array}$ & $\begin{array}{c}3.79 \\
(2.07)\end{array}$ & $\begin{array}{c}1.85 \\
(1.53)\end{array}$ \\
\hline $\mathbf{N}_{3} \mathbf{K}_{2}$ & $\begin{array}{c}2.40 \\
(1.70)\end{array}$ & $\begin{array}{c}3.73 \\
(2.06)\end{array}$ & $\begin{array}{c}1.85 \\
(1.53)\end{array}$ \\
\hline $\mathbf{N}_{3} \mathbf{K}_{3}$ & $\begin{array}{c}2.36 \\
(1.69)\end{array}$ & $\begin{array}{c}3.70 \\
(2.05)\end{array}$ & $\begin{array}{c}1.86 \\
(1.54)\end{array}$ \\
\hline 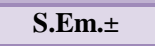 & 0.006 & 0.009 & 0.004 \\
\hline C.D. at $5 \%$ & NS & NS & NS \\
\hline
\end{tabular}


Contd.

\begin{tabular}{|c|c|c|c|}
\hline \multirow[t]{3}{*}{ Treatments } & \multicolumn{2}{|c|}{ Mirid bug (per 10 squares) } & Midge (per 10 squares) \\
\hline & \multicolumn{3}{|c|}{ 2013-14 } \\
\hline & 90 DAS & 110 DAS & 110 DAS \\
\hline $\mathbf{P}_{1} \mathbf{K}_{1}$ & $\begin{array}{c}2.41 \\
(1.71)\end{array}$ & $\begin{array}{c}3.77 \\
(2.07)\end{array}$ & $\begin{array}{c}1.85 \\
(1.53)\end{array}$ \\
\hline $\mathbf{P}_{1} \mathbf{K}_{2}$ & $\begin{array}{c}2.38 \\
(1.70)\end{array}$ & $\begin{array}{c}3.69 \\
(2.05)\end{array}$ & $\begin{array}{c}1.85 \\
(1.53)\end{array}$ \\
\hline $\mathbf{P}_{1} \mathbf{K}_{3}$ & $\begin{array}{c}2.34 \\
(1.69)\end{array}$ & $\begin{array}{c}3.65 \\
(2.04)\end{array}$ & $\begin{array}{c}1.85 \\
(1.53) \\
\end{array}$ \\
\hline $\mathbf{P}_{2} \mathbf{K}_{1}$ & $\begin{array}{c}2.39 \\
(1.70)\end{array}$ & $\begin{array}{c}3.75 \\
(2.06)\end{array}$ & $\begin{array}{c}1.86 \\
(1.54)\end{array}$ \\
\hline $\mathbf{P}_{2} \mathbf{K}_{2}$ & $\begin{array}{c}2.35 \\
(1.69)\end{array}$ & $\begin{array}{c}3.69 \\
(2.05)\end{array}$ & $\begin{array}{c}1.85 \\
(1.53) \\
\end{array}$ \\
\hline $\mathbf{P}_{2} \mathbf{K}_{3}$ & $\begin{array}{c}2.33 \\
(1.68)\end{array}$ & $\begin{array}{c}3.69 \\
(2.05)\end{array}$ & $\begin{array}{c}1.84 \\
(1.53)\end{array}$ \\
\hline S.Em. \pm & 0.005 & 0.007 & 0.004 \\
\hline C.D. at $5 \%$ & NS & NS & NS \\
\hline $\mathbf{N}_{1} \mathbf{P}_{1} \mathbf{K}_{1}$ & $\begin{array}{c}2.36 \\
(1.69)\end{array}$ & $\begin{array}{c}3.71 \\
(2.05)\end{array}$ & $\begin{array}{c}1.83 \\
(1.53)\end{array}$ \\
\hline $\mathbf{N}_{1} \mathbf{P}_{1} \mathbf{K}_{2}$ & $\begin{array}{c}2.35 \\
(1.69)\end{array}$ & $\begin{array}{c}3.65 \\
(2.04)\end{array}$ & $\begin{array}{c}1.84 \\
(1.53)\end{array}$ \\
\hline $\mathbf{N}_{1} \mathbf{P}_{1} \mathbf{K}_{3}$ & $\begin{array}{c}2.34 \\
(1.69)\end{array}$ & $\begin{array}{c}3.64 \\
(2.04)\end{array}$ & $\begin{array}{c}1.83 \\
(1.53)\end{array}$ \\
\hline $\mathbf{N}_{1} \mathbf{P}_{2} \mathbf{K}_{1}$ & $\begin{array}{c}2.35 \\
(1.69)\end{array}$ & $\begin{array}{c}3.73 \\
(2.06)\end{array}$ & $\begin{array}{c}1.86 \\
(1.54)\end{array}$ \\
\hline $\mathbf{N}_{1} \mathbf{P}_{2} \mathbf{K}_{2}$ & $\begin{array}{c}2.32 \\
(1.68)\end{array}$ & $\begin{array}{c}3.72 \\
(2.05)\end{array}$ & $\begin{array}{c}1.85 \\
(1.53) \\
\end{array}$ \\
\hline $\mathbf{N}_{1} \mathbf{P}_{2} \mathbf{K}_{3}$ & $\begin{array}{c}2.33 \\
(1.68)\end{array}$ & $\begin{array}{c}3.75 \\
(2.06)\end{array}$ & $\begin{array}{c}1.87 \\
(1.54)\end{array}$ \\
\hline $\mathbf{N}_{2} \mathbf{P}_{1} \mathbf{K}_{1}$ & $\begin{array}{c}2.41 \\
(1.71)\end{array}$ & $\begin{array}{c}3.80 \\
(2.07)\end{array}$ & $\begin{array}{c}1.87 \\
(1.54)\end{array}$ \\
\hline $\mathbf{N}_{2} \mathbf{P}_{1} \mathbf{K}_{2}$ & $\begin{array}{c}2.41 \\
(1.70)\end{array}$ & $\begin{array}{c}3.65 \\
(2.04)\end{array}$ & $\begin{array}{c}1.85 \\
(1.53)\end{array}$ \\
\hline $\mathbf{N}_{2} \mathbf{P}_{1} \mathbf{K}_{3}$ & $\begin{array}{c}2.34 \\
(1.68)\end{array}$ & $\begin{array}{c}3.60 \\
(2.02)\end{array}$ & $\begin{array}{c}1.86 \\
(1.54)\end{array}$ \\
\hline $\mathbf{N}_{2} \mathbf{P}_{2} \mathbf{K}_{1}$ & $\begin{array}{c}2.40 \\
(1.70)\end{array}$ & $\begin{array}{c}3.74 \\
(2.06)\end{array}$ & $\begin{array}{c}1.88 \\
(1.54)\end{array}$ \\
\hline $\mathbf{N}_{2} \mathbf{P}_{2} \mathbf{K}_{2}$ & $\begin{array}{c}2.32 \\
(1.68)\end{array}$ & $\begin{array}{c}3.63 \\
(2.03)\end{array}$ & $\begin{array}{c}1.86 \\
(1.54)\end{array}$ \\
\hline $\mathbf{N}_{2} \mathbf{P}_{2} \mathbf{K}_{3}$ & $\begin{array}{c}2.30 \\
(1.67)\end{array}$ & $\begin{array}{c}3.62 \\
(2.03)\end{array}$ & $\begin{array}{c}1.82 \\
(1.52)\end{array}$ \\
\hline $\mathbf{N}_{3} \mathbf{P}_{1} \mathbf{K}_{1}$ & $\begin{array}{c}2.46 \\
(1.72)\end{array}$ & $\begin{array}{c}3.80 \\
(2.07)\end{array}$ & $\begin{array}{c}1.86 \\
(1.54)\end{array}$ \\
\hline $\mathbf{N}_{3} \mathbf{P}_{1} \mathbf{K}_{2}$ & $\begin{array}{c}2.39 \\
(1.70)\end{array}$ & $\begin{array}{c}3.76 \\
(2.06)\end{array}$ & $\begin{array}{c}1.85 \\
(1.53)\end{array}$ \\
\hline $\mathbf{N}_{3} \mathbf{P}_{1} \mathbf{K}_{3}$ & $\begin{array}{c}2.35 \\
(1.69)\end{array}$ & $\begin{array}{c}3.70 \\
(2.05)\end{array}$ & $\begin{array}{c}1.88 \\
(1.54)\end{array}$ \\
\hline $\mathbf{N}_{3} \mathbf{P}_{2} \mathbf{K}_{1}$ & $\begin{array}{c}2.43 \\
(1.71)\end{array}$ & $\begin{array}{c}3.78 \\
(2.07)\end{array}$ & $\begin{array}{c}1.85 \\
(1.53)\end{array}$ \\
\hline $\mathbf{N}_{3} \mathbf{P}_{2} \mathbf{K}_{2}$ & $\begin{array}{c}2.40 \\
(1.70)\end{array}$ & $\begin{array}{c}3.71 \\
(2.05)\end{array}$ & $\begin{array}{c}1.84 \\
(1.53)\end{array}$ \\
\hline $\mathbf{N}_{3} \mathbf{P}_{2} \mathbf{K}_{3}$ & $\begin{array}{c}2.37 \\
(1.69)\end{array}$ & $\begin{array}{c}3.70 \\
(2.05)\end{array}$ & $\begin{array}{c}1.84 \\
(1.53)\end{array}$ \\
\hline S.Em. \pm & 0.009 & 0.012 & 0.006 \\
\hline C.D. at $5 \%$ & NS & NS & NS \\
\hline Control & $\begin{array}{c}2.71 \\
(1.79)\end{array}$ & $\begin{array}{c}5.12 \\
(2.37)\end{array}$ & $\begin{array}{c}1.99 \\
(1.58)\end{array}$ \\
\hline S.Em. \pm & 0.010 & 0.022 & 0.006 \\
\hline C.D. at $5 \%$ & 0.028 & $\begin{array}{r}0.062 \\
\end{array}$ & 0.018 \\
\hline Note: FYM $-5 \mathrm{t} \mathrm{ha}^{-1}$ & $\begin{array}{l}\mathrm{N}_{1}-100 \mathrm{~kg} \mathrm{ha}^{-1} \\
\mathrm{P}_{1}-50 \mathrm{~kg} \mathrm{ha}^{-1} \\
\mathrm{~K}_{1}-50 \mathrm{~kg} \mathrm{ha}^{-1} \\
\mathrm{NS}-\text { Non significant } \\
\text { Figures in the parenthes }\end{array}$ & $\begin{array}{l}\mathrm{N}_{2}-125 \mathrm{~kg} \mathrm{ha}^{-1} \\
\mathrm{P}_{2}-75 \mathrm{~kg} \mathrm{ha}^{-1} \\
\mathrm{~K}_{2}-75 \mathrm{~kg} \mathrm{ha}^{-1} \\
\text { DAS - Days after sowing }\end{array}$ & $\begin{array}{l}\mathrm{N}_{3}-150 \mathrm{~kg} \mathrm{ha}^{-1} \\
\mathrm{~K}_{3}-100 \mathrm{~kg} \mathrm{ha}^{-1}\end{array}$ \\
\hline
\end{tabular}


Significantly higher mirid bug population of 2.40 per 10 squares were recorded in the treatment $\mathrm{N}_{3}\left(150 \mathrm{~kg} \mathrm{~N} \mathrm{ha}^{-1}\right)$ at $90 \mathrm{DAS}$. The treatment receiving $\mathrm{K} @ 100 \mathrm{~kg} \mathrm{ha}^{-1}\left(\mathrm{~K}_{3}\right)$ recorded lowest mirid bug population (2.34 and 3.67 per 10 squares at 90 and 110 DAS, respectively) compared to other two levels. Different levels of NPK fertilizers neither alone nor in combination did not affect the midge population.

\section{Acknowledgement}

I would like to thank Mr. Eldad Sokolowski, Agronomist and Coordinator for China and sub-Saharan Africa/Ethiopia, International Potash Institute, Israel and Dr. S. K. Bansal, Director, Indian Potash Research Institute, Gurgaon, Haryana, for providing scholarship during my Ph. D. studies at UAS, Dharwad.

\section{References}

Ahmed, S., Habibullah Sabir, S. and Ali, C.M. 2007. Effect of different doses of nitrogen fertilizer on sucking insect pests of cotton (Gossypium hirsutum). Journal of Agricultural Research (Lahore). 45 (1): 43-48.

Ajri, D. S., Mali, A. R., Shelake, S. S., Patil, C. S. and Subedar, A. J. 1986. A status paper on problem of whitefly, Bemisia tabaci (Gennadius) in cotton and other crops in western Maharastra. Proc. Seminar on status of whitefly on Cotton, College of Agriculutre, Pune, March 14, 7p.

Altieri, M. A. and Nicholls, C. I. 2003. Soil fertility management and insect pests: harmonizing soil and plant health in agro ecosystems. Soil Tillage Research. 72: 203-211.

Amtmann, A., Troufflard, S. and Armengaud, P. 2008. The effect of potassium nutrition on pest and disease resistance in plants. Physiology Plant. 132: 682-
691.

Bi, J. L., Ballmer, G. R., Hendrix, D. L., Henneberry, T. J. and Toscano, N. C. 2001. Effect of cotton nitrogen fertilization on Bemisia argentifolii populations and honeydew production. Entomologia Experimentalis et Applicata. 99: 25-36.

Cisneros, J. J. and Godfery, L. D. 1998. Agronomic and environmental factors influencing control of cotton aphids with insecticides. In: Proc. Beltwide Cotton Conf. San Diego, California, USA, 5-9 January, pp. 1242-1246.

El-Zahi, E. S., Arif, S. A., Jehan, B. A., ElNaggar and Madeha El-Dewy, 2012. Inorganic fertilization of cotton fieldplants in relation to sucking insects and yield production components of cotton plants. Journal of American Sciences. 8 (2): 509-517.

Facknath, S. and Lalljee, B. 2005. Effect of soil-applied complex fertiliser on an insect- host plant relationship: Liriomyza trifolii on Solanum tuberosum. Entomologia Experimentalis et Applicata. 115: 6777.

Godfery, L. D., Keillor, K., Hutmacher, R. B. and Cisneros, J. J. 1999. Interaction of cotton aphid population dynamics and cotton fertilization regime in California. In: Cotton Proc. Beltwide Cotton Conf., Orlando, Florida, USA, 3-7 January, pp. 1008-1011.

Kranthi, K. R., Kranthi, S., Ramesh, K., Nagare, V. S. and Anupam Barik, 2009. Window based IRM strategies, Early sucking pest window. In: Advances in cotton IPM. Published by CICR, Nagpur, pp. 1-26.

Myers, S. W. and Gratton, C. 2006. Influence of potassium fertility on soybean aphid, Aphis glycines Matsumura (Hemiptera: Aphididae), population dynamics at a field and regional scale. 
Environmental Entomology. 35: 219227.

Nevo, E. and Coll, M. 2001. Effect of nitrogen fertilization on Aphis gossypii (Homoptera: Aphididae): variation in size, color, and reproduction. Journal of Economic Entomology. 94: 27-32.

Parihar, S. B. S. and Upadhyay, N. C. 2001. Effect of fertilizers (NPK) on incidence of leafhoppers and mite in potato crop. Insect Environment. 7: $10-11$.

Patil, B. K., Rote, N. B. and Mehta, N. P. 1986. Resurgence of sucking pests by the use of synthetic pyrethroids on cotton. In: Proc. Nat. Symp. on resurgence of sucking pests, Tamil
Nadu Agricultural University, Coimbatore, July 7-8, 1986.

Purohit, M. S. and Deshpande, A. D. 1991. Effect of inorganic fertilizers and insecticides on population density of cotton whitefly (Bemisia tabaci). Indian Journal of Agricultural Sciences. 61 (9): 696-698.

Way, M. O., Reay-jones, F. P. F., Stout, M. J. and Tarpley, L. 2006. Effects of nitrogen fertilizer applied before permanent flood on the interaction between rice and rice water weevil (Coleoptera: Curculionidae). Journal of Economic Entomology. 99: 20302037.

\section{How to cite this article:}

Jyothi, T.V., N.S. Hebsur and Parashuram Chandravanshi. 2019. Effect of Graded Levels of NPK Fertilizers on Pests Incidence in Bt Cotton in Alfisol. Int.J.Curr.Microbiol.App.Sci. 8(06): 602-616. doi: https://doi.org/10.20546/ijcmas.2019.806.070 